\title{
Hybrid Precoding for WideBand Millimeter Wave MIMO Systems in the Face of Beam Squint
}

\author{
Yun Chen, Yifeng Xiong, Da Chen, Tao Jiang, Fellow, IEEE, Soon Xin Ng, Senior Member, IEEE, \\ and Lajos Hanzo, Fellow, IEEE
}

\begin{abstract}
Hybrid Transmit Precoding (TPC) is one of the most compelling solutions for millimeter wave (mmWave) multipleinput multiple output (MIMO) systems. However, most attention has been focused on narrow-band scenarios. Hence, we dedicate our efforts to the design of hybrid TPC for wideband mmWave MIMO systems, where the beam squint dramatically affects the system performance. We firstly show that the channel matrices of the different subcarriers possess distinct subspaces in case of high bandwidths, hence traditional hybrid TPC schemes suffer from an eroded performance. Therefore, we propose novel hybrid TPC schemes exploiting the full channel state information (CSI), which project all frequencies to the central frequency and construct the common analog TPC matrix for all subcarriers. Moreover, we propose several low-complexity array-vector based hybrid TPC schemes. The high-complexity manifold optimization based hybrid TPC method and the fully digital TPC operating with and without considering beam squint are provided as benchmarks. Our extensive numerical simulations show that the proposed hybrid TPC schemes are capable of achieving similar performance to the excessive-complexity fully digital TPC, when the bandwidth tends to $0.5 \mathrm{GHz}$ and always outperform the traditional hybrid TPC schemes.
\end{abstract}

Index Terms-Millimeter wave communications, hybrid TPC, wideband, beam squint.

\section{INTRODUCTION}

M ILLIMETER wave (mmWave) multiple-input multiple output (MIMO) communication is one of the most promising next-generation techniques [1], [2]. To compensate for the high path-loss of mmWave signals so as to guarantee sufficient signal power at the receiver, typically MIMO techniques, such as Transmit Precoding (TPC) are employed. However, traditional fully digital TPC relies on a dedicated radio frequency $(\mathrm{RF})$ chain for each antenna, which imposes potentially prohibitive power consumption. To circumvent the above problem, hybrid analog/digital TPC was conceived for mmWave MIMO systems by exploiting the limited scattering

Manuscript received January 06, 2020; revised May 27, 2020 and July 25, 2020; accepted November 04, 2020. This work was supported in part by National Science Foundation of China with Grant numbers 61771216, 61831013, 61771214 and 62071195, and China Scholarship Council (CSC). L. Hanzo would like to acknowledge the financial support of the Engineering and Physical Sciences Research Council projects EP/N004558/1, EP/P034284/1, EP/P034284/1, EP/P003990/1 (COALESCE), of the Royal Society's Global Challenges Research Fund Grant as well as of the European Research Council's Advanced Fellow Grant QuantCom.

Y. Chen, D. Chen, and T. Jiang are with Wuhan National Laboratory for Optoelectronics and School of Electronic Information and Communications, Huazhong University of Science and Technology, Wuhan 430074, P. R. China (e-mail: chen_yun@hust.edu.cn; chenda@hust.edu.cn; tao.jiang@ieee.org).

Y. Xiong, S. X. Ng and L. Hanzo are with School of Electronics and Computer Science, University of Southampton, Southampton SO17 1BJ, U.K. (e-mail: yifeng.xiong@soton.ac.uk; \{sxn, lh\}@ecs.soton.ac.uk). imposed by mmWave channels [3], [4]. With the advent of the innovative techniques, hybrid TPC is capable of simultaneously transmitting multiple data streams and obtaining a similar spectral efficiency as its high-complexity fully digital TPC counterpart [5], [6].

\section{A. Prior Work}

Most prior work on constructing hybrid TPC matrices assumed a narrow-band mmWave MIMO scenario [7]-[18]. Based on the sophisticated manifold optimization, alternating minimization, matrix decomposition and iterative search methods, diverse schemes have been proposed for conceiving near-optimal hybrid TPC [7]-[10]. Low-complexity hybrid TPC schemes have enjoyed the time-light, where the sparse nature of the mmWave Channel Impulse Response (CIR) was exploited to split the channel matrix and reduce the processing dimension [11]-[13]. Improving the energy efficiency has also attracted substantial research attention, leading to attractive sub-array-connected architectures [14], switch-based solutions [15], hybrid-connection based architectures [16], and generalized sub-array-connected arrangement [17]. The beamspace based hybrid TPC design constitutes another attractive design relying on low-cost lens antenna arrays [18].

Naturally, conceiving wideband hybrid TPC designs is more challenging than that of its narrow-band counterpart, since the analog TPC is frequency-flat, while the digital TPC is frequency selective. The most direct schemes rely on searching through the same eigenvector spaces and construct the common analog TPC across all frequencies [19][21]. Alternatively, [22] and [23] defined distortion metrics for optimizing the analog TPC matrix by utilizing either a Lloyd-Max quantization based codebook or a projected gradient method, respectively. Moreover, in [7], a manifold optimization based scheme was proposed for attaining a nearoptimal solution, albeit at a high computational complexity. In [24], only statistic channel information was relied upon for constructing the analog TPC at a low feedback overhead.

Given that typically large-scale antenna arrays are used in wideband mmWave MIMO systems, the so-called spatialwideband effect causes grave beam squint [25], which represents the beam's spatial direction changes as a function of frequency. The authors of [26] considered the spatialwideband effect for the first time in massive MIMO systems and addressed the beam squint effects in MIMO transmission. For example, for a $28 \mathrm{GHz}$ central frequency and $2 \mathrm{GHz}$ bandwidth, the squint-induced angular broadening is higher 
than $20^{\circ}$ when the path angle nears $90^{\circ}$. Therefore, beam squint will dramatically affect the performance of hybrid TPC$\mathrm{s}$, since the analog TPC fails to resolve different frequencies and having limited number of radio frequency $(\mathrm{RF})$ chains also limits the ability of the digital TPC to do so.

Yet, only few contributions have taken the beam squint into account for the hybrid TPC architecture [27]-[31]. In [27], the beam squint vs frequency was derived and a hybrid scheme was proposed for channel estimation in wideband mmWave systems. In [28], the authors pointed out that the wideband mmWave channel is not sparse in the angle-delay domain and designed a window-based solution to concentrate the energy of each multipath component. In [29], a wideband hybrid TPC scheme was proposed for a lens-antenna array based architecture by capturing the power from multiple focusedenergy beams. In [30], the high-complexity iterative manifold optimization method was utilized for designing hybrid TPC in the face of beam squint. Additionally, by exploiting the common dominant subspaces across all frequencies, several compressive subspace estimation based frequency-flat hybrid TPC schemes were conceived in [31]. However, the authors of [31] only considered two special cases, where both the system bandwidth and the number of antennas were limited, and the number of paths was equal to the number of RF chains.

\section{B. Contributions}

Against the background, we focus our attention on the challenging design of hybrid TPCs for wideband mmWave MIMO systems subject to beam squint. By fully exploiting the angular domain properties of wideband mmWave channels, we propose several novel hybrid TPC designs by either exploiting the full channel state information (CSI) across the entire frequency range or from the array vectors' perspectives. These sophisticated methods provide design guidelines for powerful wideband mmWave systems. Our main contributions are listed as follows:

- We show that the channel matrices of the different subcarriers possess distinct subspaces in case of high bandwidths, hence the total mmWave channel is not sparse when constructing the analog TPC and traditional hybrid TPC schemes are unable to achieve close performance to its fully digital TPC counterpart. This is the inherent disadvantage of the hybrid TPC architecture, given that the analog TPC can only be applied in the time domain. Several polar diagrams are firstly provided for exemplifying the phases/angles of the paths, where all frequencies are projected to the central frequency. It can be observed from the polar diagrams that the path angles are spread from the central frequency and the closer the angle in terms of its central frequency to the end side, the larger the angular spread becomes. Moreover, we define the mean chordal distance (MCD) between the subspaces of the central channel matrix (defined in Section III) and of the channel matrices on all subcarriers as a potent metric of quantifying the effect of beam squint on the subspaces of the wideband mmWave channel. Then, we further define the average achievable rate gap
(AARG) encountered for quantifying the performance loss encountered upon applying the subspaces of the central channel matrix for constructing the analog TPC for all subcarriers. The corresponding simulation results show that both the MCD and the AARG increase with the bandwidth and with the dimension of the subspaces. This indicates that traditional frequency-flat analog TPC schemes cannot meet the requirement of the wideband mmWave MIMO systems having large bandwidths.

- We propose several hybrid TPC schemes for wideband mmWave scenarios, which are based on the full CSI spanning across all frequencies. Specifically, we first regard the channel matrices of all subcarriers as a nonsparse single "frequency-flat" channel so that we can simultaneously construct the analog TPC matrix for all subcarriers, which further inspires us to propose mean channel matrix (MCM) and mean channel-covariance matrix (MCCM) based hybrid TPC schemes. Then, we extend the extrinsic mean of subspaces (EMS) based method provided in [31], and propose a generalized EMS (GEMS) based hybrid TPC scheme, where both the number of RF chains and the number of paths can be arbitrary, whilst avoiding the power leakage to unused subspaces.

- We propose several low-complexity array-vector based hybrid TPC schemes for wideband mmWave MIMO scenarios. The core of these schemes is to construct a widebeam for mitigating the effect of beam squint. Firstly, by relying on multiple sets of phase shifters (PSs), a scheme combining the beams of all subcarriers related to the same path is proposed, which has the maximum beam width. We then conceive a scheme that only choose the beams of the central subcarrier as a low-complexity benchmark, which has the narrowest beam width but the highest peak beam gain. Bearing in mind the trade-off between the beam width and beam gain, both of which affect the performance, we also propose to combine beams of partial subcarriers so as to increase the achievable rate.

- Extensive numerical simulations are provided for evaluating the performance of our proposed hybrid $\mathrm{T}$ PC schemes. Our simulation results demonstrate that the proposed wideband hybrid TPC schemes are capable of achieving similar performance to the excessivecomplexity fully digital TPC scheme, when the bandwidth tends to $0.5 \mathrm{GHz}$. They also always outperform the traditional hybrid TPC schemes that do not take the beam squint into account, when considering bandwidths between 0.3 and $4 \mathrm{GHz}$. Moreover, we also observe that our proposed schemes benefit from increasing the number of RF chains, and performance well even for $4 \mathrm{GHz}$ bandwidth, substantially outperforming the traditional hybrid TPC schemes.

The rest of the paper is organized as follows. Section II presents our system model, channel model, problem formulation and some of our assumptions. The properties of wideband mmWave channels are introduced in Section III. In Section IV, our full CSI based hybrid TPC schemes are proposed. Section 
$\mathrm{V}$ introduces the proposed low-complexity array-vector based hybrid TPC schemes. Section VI discusses our simulation results. Finally, we conclude in Section VII.

The notations are summarized in Table I.

\section{System Model, Channel Model and Problem FORMULATION}

In this section, the system model, the wideband mmWave channel model, the problem formulation and some of our assumptions are introduced.

\section{A. System Model}

Consider a orthogonal frequency-division multiplexing (OFDM) modulation aided wideband mmWave MIMO system shown in Fig. 1. The base station (BS) is equipped with $N_{\mathrm{t}}$ antennas and $N_{\mathrm{RF}}^{\mathrm{t}} \mathrm{RF}$ chains. At the receiver, there are $N_{\mathrm{r}}$ receiver antennas and $N_{\mathrm{RF}}^{\mathrm{r}} \mathrm{RF}$ chains. We consider the fully-connected hybrid TPC architecture at both the BS and the receiver, where each RF chain is connected to all antennas through multiple sets of PSs. Due to the inherent hardware constraint on the hybrid TPC architecture, we have $N_{\mathrm{s}} \leq N_{\mathrm{RF}}^{\mathrm{t}} \leq N_{\mathrm{t}}$ and $N_{\mathrm{s}} \leq N_{\mathrm{RF}}^{\mathrm{r}} \leq N_{\mathrm{r}}$, where $N_{\mathrm{s}}$ is the number of data streams.

At the BS, the $N_{\mathrm{s}}$ data streams conveyed by each subcarrier are first precoded by an $\left(N_{\mathrm{RF}}^{\mathrm{t}} \times N_{\mathrm{s}}\right)$-element digital TPC matrix $\mathbf{F}_{\mathrm{BB}}[k], k=1,2, \ldots, K$, where $K$ is the total number of subcarriers. Then, following $K$-point IFFT modulation and adding the CP, the signals are precoded by the $\left(N_{\mathrm{t}} \times N_{\mathrm{RF}}^{\mathrm{t}}\right)$ element analog TPC matrix $\mathbf{F}_{\mathrm{RF}}$. The constant modulus (CM) constraint is imposed according to $\left|\left[\mathbf{F}_{\mathrm{RF}}\right]_{i, j}\right|^{2}=\frac{1}{N_{\mathrm{t}}}$ due to utilizing PSs. Therefore, the discrete-time transmitted signal vector at the $k^{t h}$ subcarrier is

$$
\mathbf{x}[k]=\mathbf{F}_{\mathrm{RF}} \mathbf{F}_{\mathrm{BB}}[k] \mathbf{s}[k],
$$

where $\mathbf{s}[k]$ is the $\left(N_{\mathrm{s}} \times 1\right)$-element transmit signal vector at the $k^{t h}$ subcarrier. Furthermore, we have $\mathbb{E}[\mathbf{s}[k]]=0$ and $\mathbb{E}\left[\mathbf{s}[k] \mathbf{s}^{*}[k]\right]=\mathbf{I}_{N_{\mathrm{s}}}$. Please note that the digital TPC $\mathbf{F}_{\mathrm{BB}}[k]$ is frequency selective, while the analog TPC $\mathbf{F}_{\mathrm{RF}}$ is frequencyflat, hence the latter has the same effect on all subcarriers. This is one of the main distinguishing characteristics of the wideband hybrid TPC compared to the conventional fullydigital TPC. However, this feature limits the performance of the hybrid TPC in wideband systems, which is due to the deleterious effects of beam squint.

At the receiver, we assume perfect clock and carrier frequency synchronization and the $\mathrm{CP}$ is removed. Then the discretetime signals received at the $k^{\text {th }}$ subcarrier after $K$-point FFT can be formulated as

$$
\mathbf{y}[k]=\mathbf{W}_{\mathrm{BB}}^{*}[k] \mathbf{W}_{\mathrm{RF}}^{*} \mathbf{H}[k] \mathbf{x}[k]+\mathbf{n}[k],
$$

where $\mathbf{W}_{\mathrm{RF}}$ is the $\left(N_{\mathrm{r}} \times N_{\mathrm{RF}}^{\mathrm{r}}\right)$-element $\mathrm{RF}$ combining matrix and $\mathbf{W}_{\mathrm{BB}}[k]$ is the $\left(N_{\mathrm{RF}}^{\mathrm{r}} \times N_{\mathrm{s}}\right)$-element digital combining matrix at the $k^{t h}$ subcarrier. Moreover, $\mathbf{H}[k]$ is the channel matrix at the $k^{\text {th }}$ subcarrier, and $\mathbf{n}[k] \sim \mathcal{C N}\left(0, \sigma_{n}^{2} \mathbf{I}_{N_{\mathrm{r}}}\right)$ is the additive white Gaussian noise vector. Equal power sharing is assumed for the subcarriers similar to [21], [23]. Thus we have $\left\|\mathbf{F}_{\mathrm{RF}} \mathbf{F}_{\mathrm{BB}}[k]\right\|_{F}^{2}=\frac{P_{\mathrm{t}}}{N_{\mathrm{s}}}$, where $P_{\mathrm{t}}$ is the total transmit power.

\section{B. Channel Model}

Due to the short wavelength of the mmWave signals, they experience severe path loss, which leads to limited scattering. Therefore, the Saleh-Valenzuela model is adopted for modelling the wideband mmWave channel [4]. Assuming that the channel consists of $N_{\mathrm{cl}}$ clusters and each cluster contributes $N_{\text {ray }}$ propagation paths, the frequency domain mmWave channel matrix at the $k^{\text {th }}$ subcarrier can be formulated as [21]

$$
\mathbf{H}[k]=\gamma \sum_{m=1}^{N_{\mathrm{cl}}} \sum_{n=1}^{N_{\text {ray }}} \alpha_{m, n} \beta_{m, n, k} \mathbf{a}_{\mathrm{r}}\left(\phi_{m, n, k}\right) \mathbf{a}_{\mathrm{t}}^{*}\left(\varphi_{m, n, k}\right),
$$

where $\gamma=\sqrt{N_{\mathrm{t}} N_{\mathrm{r}} / N_{\mathrm{cl}} N_{\text {ray }}}$ is the normalization factor, $\alpha_{m, n}$ denotes the path gains, $\beta_{m, n, k}=e^{-j 2 \pi \tau_{m, n} f_{k}}$ represents the delay component, in which $\tau_{m, n}$ is the delay of the $n^{t h}$ path in the $m^{t h}$ cluster. We consider a uniform linear array (ULA), but the proposed schemes are applicable to arbitrary antenna arrays, such as uniform planar arrays (UPA). Therefore, the array response vectors $\mathbf{a}_{\mathrm{r}}\left(\phi_{m, n, k}\right)$ and $\mathbf{a}_{\mathrm{t}}\left(\phi_{m, n, k}\right)$ can be written as

$$
\mathbf{a}_{\mathrm{r}}\left(\phi_{m, n, k}\right)=\frac{1}{\sqrt{N_{\mathrm{r}}}}\left[1, e^{j 2 \pi \phi_{m, n, k}}, \ldots, e^{j\left(N_{\mathrm{r}}-1\right) 2 \pi \phi_{m, n, k}}\right]^{T},
$$

and

$$
\mathbf{a}_{\mathrm{t}}\left(\varphi_{m, n, k}\right)=\frac{1}{\sqrt{N_{\mathrm{t}}}}\left[1, e^{j 2 \pi \varphi_{m, n, k}}, \ldots, e^{j\left(N_{\mathrm{t}}-1\right) 2 \pi \varphi_{m, n, k}}\right]^{T},
$$

respectively. Note that (3) can be presented in a more compact yet equivalent form as

$$
\mathbf{H}[k]=\gamma \mathbf{A}_{\mathrm{r}}[k] \boldsymbol{\Delta}[k] \mathbf{A}_{\mathrm{t}}^{*}[k],
$$

where we have:

$$
\begin{aligned}
\boldsymbol{\Delta}[k] & =\boldsymbol{\Delta}_{\alpha} \boldsymbol{\Delta}_{\beta}[k] \\
& =\left[\begin{array}{c}
\alpha_{1,1} \\
\alpha_{1,2} \\
\ddots \\
\alpha_{N_{\mathrm{cl}}, N_{\mathrm{ray}}}
\end{array}\right]\left[\begin{array}{c}
\beta_{1,1, k} \\
\beta_{1,2, k} \\
\ddots \\
\beta_{N_{\mathrm{cl}}, N_{\mathrm{ray}}, k}
\end{array}\right] \\
& {\left[\begin{array}{c}
\alpha_{1,1} \beta_{1,1, k} \\
\alpha_{1,2} \beta_{1,2, k} \\
\ddots \\
\alpha_{N_{\mathrm{cl}}, N_{\mathrm{ray}}} \beta_{N_{\mathrm{cl}}, N_{\mathrm{ray}}, k}
\end{array}\right], }
\end{aligned}
$$

$$
\mathbf{A}_{\mathrm{r}}[k]=\left[\mathbf{a}_{\mathrm{r}}\left(\phi_{1,1, k}\right), \ldots, \mathbf{a}_{\mathrm{r}}\left(\phi_{1, N_{\mathrm{ray}}, k}\right), \ldots, \mathbf{a}_{\mathrm{r}}\left(\phi_{N_{\mathrm{cl}}, N_{\text {ray }}, k}\right)\right],
$$

and

$$
\mathbf{A}_{\mathrm{t}}[k]=\left[\mathbf{a}_{\mathrm{t}}\left(\varphi_{1,1, k}\right), \ldots, \mathbf{a}_{\mathrm{t}}\left(\varphi_{1, N_{\mathrm{ray}}, k}\right), \ldots, \mathbf{a}_{\mathrm{t}}\left(\varphi_{N_{\mathrm{cl}}, N_{\mathrm{ray}}, k}\right)\right] .
$$

In (4) and (5) $\phi_{m, n, k}$ and $\varphi_{m, n, k}$ denote the spatial direction (phase) of the $n^{t h}$ path in the $m^{t h}$ cluster at the $k^{t h}$ subcarrier, respectively, which can be presented as [32]

$$
\begin{aligned}
& \phi_{m, n, k}=\frac{f_{k}}{\mathrm{c}} d \sin \left(\theta_{m, n}\right), \\
& \varphi_{m, n, k}=\frac{f_{k}}{\mathrm{c}} d \sin \left(\vartheta_{m, n}\right),
\end{aligned}
$$


TABLE I

NOTATIONS.

\begin{tabular}{|l|l|l|l|}
\hline$a$ & a scalar & $\|\mathbf{A}\|_{F}$ & the Frobenius norm of $\mathbf{A}$ \\
\hline $\mathbf{a}$ & a vector & {$[\mathbf{A}]_{i, j}$} & the $(i, j)^{t h}$ element of $\mathbf{A}$ \\
\hline $\mathbf{A}$ & a matrix & $\measuredangle(\mathbf{A})$ & a matrix whose $(i, j)^{t h}$ element equals to the phase of the $(i, j)^{t h}$ element in $\mathbf{A}$ \\
\hline $\mathcal{A}$ & a set & $\mathcal{C N}(\mathbf{a}, \mathbf{A})$ & a complex Gaussian vector with mean a and covariance matrix $\mathbf{A}$ \\
\hline$|\mathbf{a}|$ & the modulus of $\mathbf{a}$ & $\mathbf{I}_{N}$ & a $N \times N$ identity matrix \\
\hline$|\mathbf{A}|$ & the determinant of $\mathbf{A}$ & $\mathbb{E}[\mathbf{A}]$ & the expectation of $\mathbf{A}$ \\
\hline $\mathbf{A}^{T}$ & the transpose of $\mathbf{A}$ & $\lceil\cdot\rceil$ & ceiling integer function \\
\hline $\mathbf{A}^{*}$ & the conjugate transpose of $\mathbf{A}$ & $\lfloor\cdot\rfloor$ & floor integer function \\
\hline $\mathbf{A}^{-1}$ & the inverse of $\mathbf{A}$ & & \\
\hline
\end{tabular}
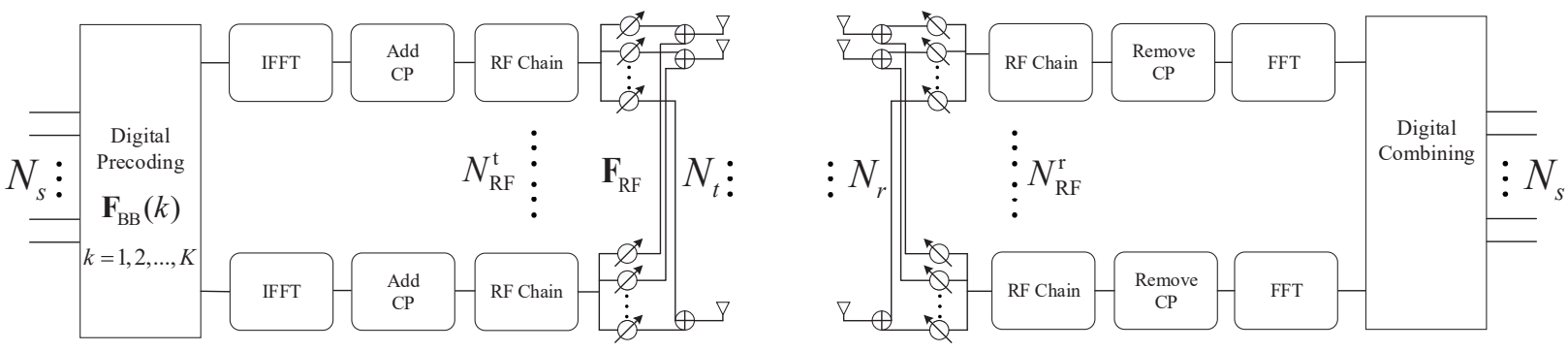

Fig. 1. The block diagram of the wideband multiuser mmWave MIMO system model that employs the hybrid TPC.

where $f_{k}=f_{\mathrm{c}}+\frac{f_{\mathrm{s}}}{K}\left(k-1-\frac{K-1}{2}\right)$ is the frequency at the $k^{t h}$ subcarrier, $f_{\mathrm{c}}$ and $f_{\mathrm{s}}$ are the carrier frequency and bandwidth, respectively, while $\theta_{m, n} \in[0,2 \pi)$ and $\vartheta_{m, n} \in[0,2 \pi)$ are the corresponding physical angle of arrival (AOA) and angle of departure (AOD) at the receiver and the transmitter, respectively. Furthermore, c represents the speed of light and $d=\frac{\mathrm{c}}{2 f_{\mathrm{c}}}=\frac{\lambda}{2}$ denotes the antenna-element spacing. Note that $\lambda$ is the signal wavelength of the central frequency. It is usually assumed in the narrow-band scenario that $f_{k}=f_{\mathrm{c}}$ and the spatial directions are frequency-independent, i,e., $\phi_{m, n, k}=\frac{1}{2} \sin \left(\theta_{m, n}\right)$ and $\varphi_{m, n, k}=\frac{1}{2} \sin \left(\vartheta_{m, n}\right)$. However, in the wideband mmWave MIMO system, $\phi_{m, n, k}$ and $\varphi_{m, n, k}$ are frequency-dependent due to the strong beam squint. Thus the narrowband scenario1's frequency-independence assumption no longer holds. We will detail the impact of beam squint on the wideband mmWave channel in Section III.

\section{Problem Formulation}

In this paper we mainly focus our attention on the design of hybrid TPCs at the BS and assume that the receiver is capable of performing optimal nearest neighbor detection based on the $N_{\mathrm{r}}$-dimensional received signal relying on a fully digital receiver-combining architecture, similar to [21], [22]. Therefore, the achievable sum rate is adopted as our system performance metric, whilst assuming Gaussian signalling over the wideband mmWave channel, yielding:

$$
\begin{aligned}
R_{\text {sum }}=\frac{1}{K} \sum_{k=1}^{K} \log _{2}( & \mid \mathbf{I}_{N_{\mathrm{r}}}+\frac{1}{\sigma_{n}^{2}}\left(\mathbf{H}[k] \mathbf{F}_{\mathrm{RF}} \mathbf{F}_{\mathrm{BB}}[k]\right. \\
& \left.\left.\times \mathbf{F}_{\mathrm{BB}}^{*}[k] \mathbf{F}_{\mathrm{RF}}^{*} \mathbf{H}^{*}[k]\right) \mid\right) .
\end{aligned}
$$

Accordingly, the optimization problem can be formulated as

$$
\begin{array}{r}
\left(\mathbf{F}_{\mathrm{RF}}^{\mathrm{opt}}, \mathbf{F}_{\mathrm{BB}}^{\mathrm{opt}}[k]\right)=\arg \max R_{\mathrm{sum}}, \forall k, \\
\text { s.t. }\left|\left[\mathbf{F}_{\mathrm{RF}}\right]_{i, j}\right|^{2}=\frac{1}{N_{\mathrm{t}}}, \forall i, j, \\
\\
\quad\left\|\mathbf{F}_{\mathrm{RF}} \mathbf{F}_{\mathrm{BB}}[k]\right\|_{F}^{2}=\frac{P_{\mathrm{t}}}{N_{\mathrm{s}}} .
\end{array}
$$

However, it is challenging to directly solve (13) due to the nonconvex CM constraint imposed on the analog TPC elements and the power constraint imposed on the precoders, which requires the joint optimization of the analog and digital TPCs as well as a complex iterative process for finding the globally optimal solution. We circumvent this challenge by separating the construction of the analog and digital TPCs to facilitate a low-complexity design in line with the state-of-the-art [8], [21]-[23]. This decoupled manner is still capable of achieving a high performance, whilst circumventing complex iterative operations. Moreover, in the design of the digital TPC, perfect CSI knowledge is assumed at the BS. Once the analog TPC has been constructed, the digital TPC matrix matched to each subcarrier can be directly obtained using the following lemma.

Lemma 1: Define a dummy effective channel matrix after the analog TPC $\widehat{\mathbf{H}}[k]$ as

$$
\widehat{\mathbf{H}}[k]=\mathbf{H}[k] \mathbf{F}_{\mathrm{RF}}\left(\mathbf{F}_{\mathrm{RF}}^{*} \mathbf{F}_{\mathrm{RF}}\right)^{-\frac{1}{2}} .
$$

Then the corresponding SVD decomposition of the dummy correlation matrix $\widehat{\mathbf{R}}[k]$ is given by

$$
\widehat{\mathbf{R}}[k]=\widehat{\mathbf{H}}^{*}[k] \widehat{\mathbf{H}}[k]=\widehat{\mathbf{V}}[k] \widehat{\boldsymbol{\Sigma}}[k] \widehat{\mathbf{V}}^{*}[k] .
$$


Then, the optimal digital TPC $\mathbf{F}_{\mathrm{BB}}[k]$ of the $k^{t h}$ subcarrier can be formulated as

$$
\mathbf{F}_{\mathrm{BB}}[k]=\left(\mathbf{F}_{\mathrm{RF}}^{*} \mathbf{F}_{\mathrm{RF}}\right)^{-\frac{1}{2}} \widehat{\mathbf{V}}[k]\left(:, 1: N_{\mathrm{s}}\right),
$$

where $\widehat{\mathbf{V}}[k]\left(:, 1: N_{\mathrm{s}}\right)$ consists of the first $N_{\mathrm{s}}$ columns of $\widehat{\mathbf{V}}[k]$.

Proof: To circumvent the problem of power-coupling between the different TPCs, we first let $\mathbf{F}_{\mathrm{BB}}[k]=$ $\left(\mathbf{F}_{\mathrm{RF}}^{*} \mathbf{F}_{\mathrm{RF}}\right)^{-\frac{1}{2}} \widehat{\mathbf{F}}_{\mathrm{BB}}[k]$. Then the problem (13) becomes equivalent to

$$
\begin{aligned}
&\left(\mathbf{F}_{\mathrm{BB}}^{\mathrm{opt}}[k]\right)= \arg \max \widetilde{R}_{\mathrm{sum}}, \forall k, \\
& \text { s.t. }\left\|\widehat{\mathbf{F}}_{\mathrm{BB}}[k]\right\|_{F}^{2}=\frac{P_{\mathrm{t}}}{N_{\mathrm{s}}},
\end{aligned}
$$

and $\widetilde{R}_{\text {sum }}$ can be written as

$$
\begin{aligned}
\widetilde{R}_{\text {sum }}=\frac{1}{K} \sum_{k=1}^{K} \log _{2} & \left(\mid \mathbf{I}_{N_{\mathrm{r}}}+\frac{1}{\sigma_{n}^{2}}\left(\widehat{\mathbf{H}}[k] \widehat{\mathbf{F}}_{\mathrm{BB}}[k]\right.\right. \\
& \left.\left.\times \widehat{\mathbf{F}}_{\mathrm{BB}}^{*}[k] \widehat{\mathbf{H}}^{*}[k]\right) \mid\right) .
\end{aligned}
$$

Since $\widehat{\mathbf{H}}[k]$ is known at the transmitter and the power is now decoupled, the optimal $\widehat{\mathbf{F}}_{\mathrm{BB}}[k]$ can be readily constructed by the SVD of the $\left(N_{\mathrm{RF}} \times N_{\mathrm{RF}}\right)$-element dummy correlation matrix $\widehat{\mathbf{R}}[k]$, i.e., $\widehat{\mathbf{F}}_{\mathrm{BB}}[k]=\widehat{\mathbf{V}}[k]\left(:, 1: N_{\mathrm{s}}\right)$ and we can obtain the result shown in (16).

Therefore, in the following we will mainly focus our attention on the design of the analog TPC, which is based on the full CSI in Section IV and only based on the array-vectors in order to facilitate the low-complexity design to be outlined in Section V. The corresponding optimization problem now becomes

$$
\begin{aligned}
\left(\mathbf{F}_{\mathrm{RF}}^{\mathrm{opt}}\right)= & \arg \max R_{\mathrm{sum}}, \\
& \text { s.t. }\left|\left[\mathbf{F}_{\mathrm{RF}}\right]_{i, j}\right|^{2}=\frac{1}{N_{\mathrm{t}}}, \forall i, j .
\end{aligned}
$$

Note that the power constraint can be satisfied by power normalization after the digital TPC as $\mathbf{F}_{\mathrm{BB}}[k]=$ $\sqrt{\frac{P_{\mathrm{t}}}{N_{\mathrm{s}}}} \frac{\mathbf{F}_{\mathrm{BB}}[k]}{\left\|\mathbf{F}_{\mathrm{RF}} \mathbf{F}_{\mathrm{BB}}[k]\right\|_{F}}$.

\section{PRoperties of ANGULAR DOMAIN WIDEBAND MMWAVE CHANNELS}

To begin our analog TPC design, we will first introduce the wideband mmWave channel properties in the time-angular domain, which is based on polar diagrams and our MCD and AARG metrics.

According to (11) and taking into account the equivalent physical steering $\operatorname{AoD} \vartheta_{m, n, k}$ of the $k^{t h}$ subcarrier, we have

$$
\frac{f_{\mathrm{c}}}{\mathrm{c}} d \sin \left(\vartheta_{m, n, k}\right)=\frac{f_{k}}{\mathrm{c}} d \sin \left(\vartheta_{m, n}\right) \pm z,
$$

where $z \in \mathbb{Z}$ due to the periodic nature of the phase, which limits the range of phases to $[-1,1]$. Therefore, $\vartheta_{m, n, k}$ can be expresed as

$$
\begin{aligned}
& \vartheta_{m, n, k} \\
& =\arcsin \left(\frac{f_{k}}{f_{\mathrm{c}}} \sin \left(\vartheta_{m, n}\right) \pm 2 z\right) \\
& =\arcsin \left(\left(1+\frac{f_{\mathrm{s}}}{f_{\mathrm{c}} K}\left(k-1-\frac{K-1}{2}\right)\right) \sin \left(\vartheta_{m, n}\right) \pm 2 z\right)
\end{aligned}
$$

The polar plot Fig. 2 shows the phases/angles relative to the broadside for the paths of all subcarriers, where all frequencies are projected to the central frequency. This implies that the array vectors of different subcarrier frequencies are projected to the quantization codebook (can be found in [6]) constructed at the central frequency. We observe that when the bandwidth increases, the angular spread becomes non-negligible and the channel is no longer sparse in the time-angular domain, despite the fact that there is only three dominant paths. Moreover, when the angle is closer to $90^{\circ}$, the squint-induced angular broadening is larger, which may exceed $20^{\circ}$ when considering a $2 \mathrm{GHz}$ bandwidth and $28 \mathrm{GHz}$ carrier.

To further underline the difference in terms of the channel matrix subspaces caused by beam squint, we define the MCD between the effective subspaces (the subspaces generated by the first $N_{\mathrm{s}}$ singular vectors) of the central channel matrix and of the channel matrices on all subcarriers as an effective metric. Firstly, the wideband channel without considering the beam squint is defined as the central channel, which is given by

$$
\mathbf{H}_{\mathrm{cent}}[k]=\gamma \sum_{m=1}^{N_{\mathrm{cl}}} \sum_{n=1}^{N_{\mathrm{ray}}} \alpha_{m, n} \beta_{m, n, k} \mathbf{a}_{\mathrm{r}}\left(\bar{\phi}_{m, n}\right) \mathbf{a}_{\mathrm{t}}^{*}\left(\bar{\varphi}_{m, n}\right),
$$

where $\bar{\varphi}_{m, n}=\frac{1}{2} \sin \left(\vartheta_{m, n}\right)$ and $\bar{\phi}_{m, n}=\frac{1}{2} \sin \left(\theta_{m, n}\right)$, and the corresponding SVD is formulated as:

$$
\mathbf{H}[k]_{\text {cent }}=\mathbf{U}_{\text {cent }} \boldsymbol{\Sigma}_{\text {cent }}[k] \mathbf{V}_{\text {cent }}^{*} .
$$

Note that the singular vectors of the different frequencies in the central channel are the same, but the singular values are different, since each frequency has different delay components. Then, the SVD of each subcarrier signal can be written as

$$
\mathbf{H}[k]=\mathbf{U}[k] \boldsymbol{\Sigma}[k] \mathbf{V}^{*}[k], k=1,2, \ldots, K .
$$

Therefore, the MCD can be formulated as

$$
\begin{aligned}
& \text { MCD } \\
& =\frac{1}{K} \sum_{k=1}^{K} \operatorname{dist}_{\text {chord }}\left(\mathcal{V}_{\text {cent }}^{N_{\mathrm{s}}}, \mathcal{V}^{N_{\mathrm{s}}}[k]\right) \\
& =\frac{1}{K} \sum_{k=1}^{K}\left[N_{\mathrm{s}}-\left\|\mathbf{V}_{\text {cent }}^{*}\left(:, 1: N_{\mathrm{s}}\right) \mathbf{V}\left(:, 1: N_{\mathrm{s}}\right)[k]\right\|_{F}^{2}\right]^{\frac{1}{2}},
\end{aligned}
$$

where the columns of $\mathbf{V}_{\text {cent }}\left(:, 1: N_{\mathrm{s}}\right)$ and $\mathbf{V}\left(:, 1: N_{\mathrm{s}}\right)[k]$ form orthogonal bases for the subspaces $\mathcal{V}_{\text {cent }}^{N_{\mathrm{s}}}[k]$ and $\mathcal{V}^{N_{\mathrm{s}}}[k]$, respectively. Fig. 3 shows the MCD for different bandwidths and different effective subspace dimensions (i.e., the number of data streams $N_{\mathrm{s}}$ ). It can be observed that the MCD increases both with the bandwidth and with $N_{\mathrm{s}}$. Moreover, the normalized MCDs $\left(\mathrm{MCDs} / \sqrt{N_{\mathrm{s}}}\right)$ are higher than 0.5 


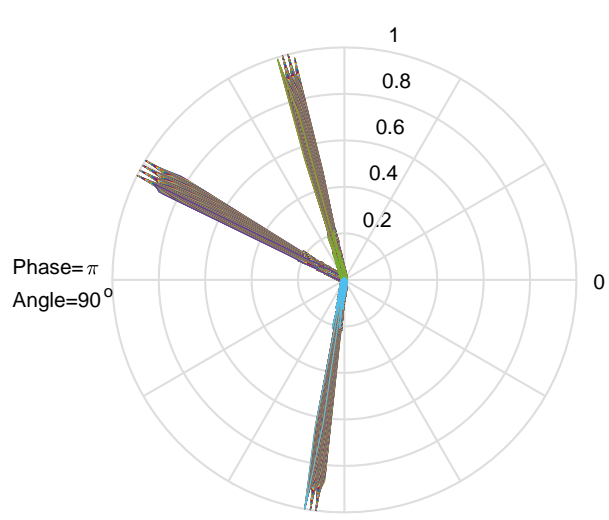

(a)

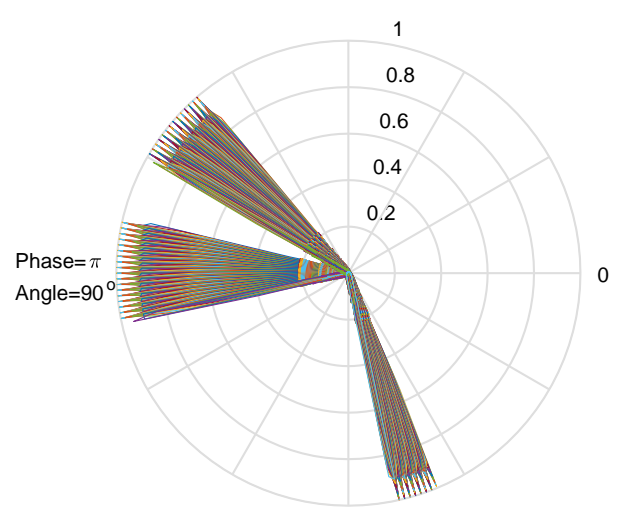

(b)

Fig. 2. An example of the polar plot of the paths in wideband mmWave channels with (a) $1 \mathrm{GHz}$ bandwidth and (b) $4 \mathrm{GHz}$ bandwidth, where $f_{\mathrm{c}}=28$ $\mathrm{GHz}, N_{\mathrm{t}}=128, K=128, N_{\mathrm{cl}}=3, N_{\text {ray }}=1$ and the number of quantization bits $b$ is set to 8 .

for different numbers of data streams, when the bandwidth is as high as $4 \mathrm{GHz}$. Therefore, the assumption that the matrices of different frequencies share a common subspaces no longer holds. Furthermore, we also quantify the AARG when applying the central channel to construct the hybrid TPC for all subcarries, which is defined as

$$
\begin{aligned}
& R_{\mathrm{AARG}} \\
& =\frac{1}{K} \sum_{k=1}^{K} R[k]-R_{\text {cent }}[k] \\
& =\frac{1}{K} \sum_{k=1}^{K} \log _{2}\left(\left|\mathbf{I}_{N_{\mathrm{r}}}+\frac{1}{\sigma_{n}^{2}}\left(\mathbf{H}[k] \mathbf{F}[k] \mathbf{F}^{*}[k] \mathbf{H}^{*}[k]\right)\right|\right) \\
& \quad-\log _{2}\left(\left|\mathbf{I}_{N_{\mathrm{r}}}+\frac{1}{\sigma_{n}^{2}}\left(\mathbf{H}[k] \mathbf{F}^{\mathrm{cent}}[k]\left(\mathbf{F}^{\mathrm{cent}}[k]\right)^{*} \mathbf{H}^{*}[k]\right)\right|\right),
\end{aligned}
$$

where we have $\mathbf{F}[k]=\mathbf{F}_{\mathrm{RF}}[k] \mathbf{F}_{\mathrm{BB}}[k], \quad \mathbf{F}^{\text {cent }}[k]=$ $\mathbf{F}_{\mathrm{RF}}^{\mathrm{cent}} \mathbf{F}_{\mathrm{BB}}^{\mathrm{cent}}[k]$. To elaborate, $\mathbf{F}_{\mathrm{RF}}[k]$ and $\mathbf{F}_{\mathrm{RF}}^{\mathrm{cent}}$ are constructed

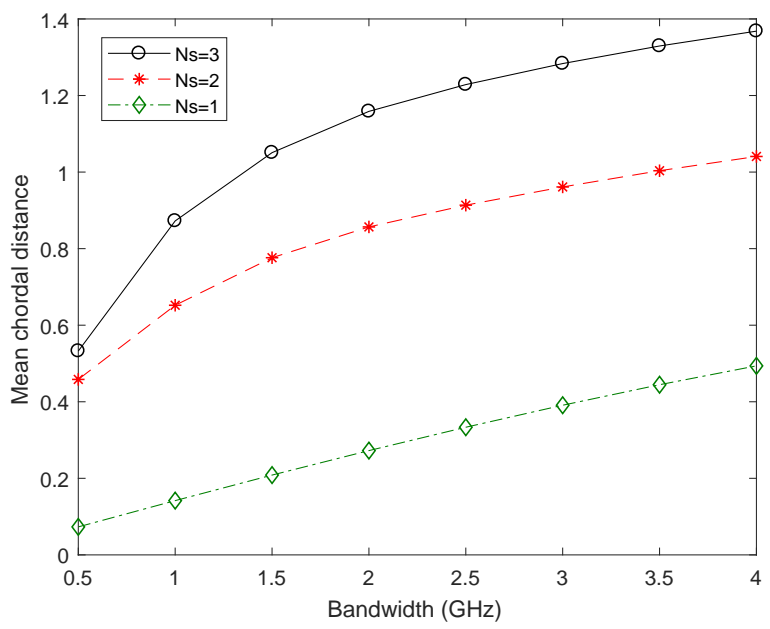

Fig. 3. An example of the MCD between the subspaces of the central channel and of the channel on each subcarrier for different numbers of data streams, where $f_{\mathrm{c}}=28 \mathrm{GHz}, N_{\mathrm{t}}=128, N_{\mathrm{r}}=16, K=128, N_{\mathrm{cl}}=3, N_{\text {ray }}=1$.

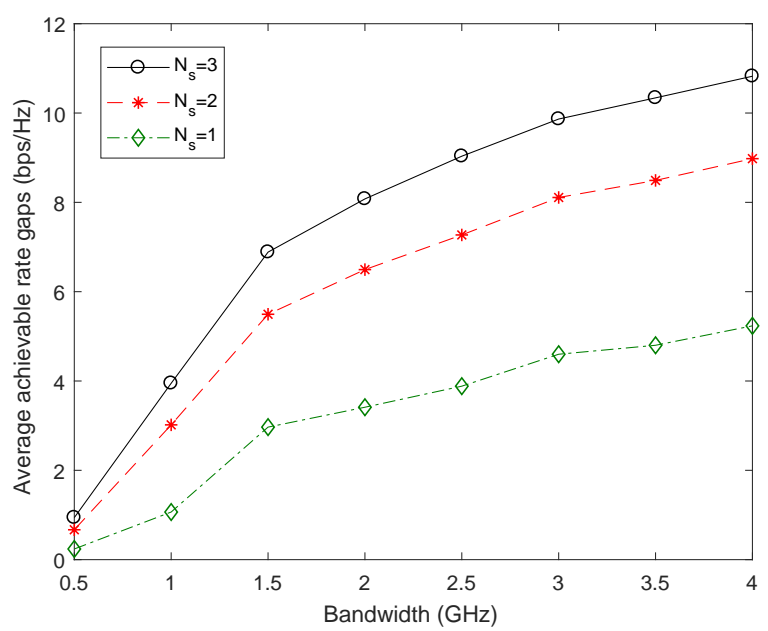

Fig. 4. An example of the AARG between the scheme that applies the central channel to construct the hybrid TPC for all subcarriers and the scheme that applies each subcarrier channel to construct the hybrid TPC for each subcarrier, where $f_{\mathrm{c}}=28 \mathrm{GHz}, N_{\mathrm{t}}=128, N_{\mathrm{r}}=16, K=128, N_{\mathrm{RF}}^{\mathrm{t}}=$ $N_{\mathrm{s}}, N_{\mathrm{cl}}=3, N_{\text {ray }}=1$.

by phase exaction from the corresponding subspace vectors as follows:

$$
\begin{gathered}
{\left[\mathbf{F}_{\mathrm{RF}}[k]\right]_{i, j}=\frac{1}{\sqrt{N_{\mathrm{t}}}} e^{j \measuredangle\left(\left[\mathbf{V}\left(:, 1: N_{\mathrm{RF}}\right)[k]\right]_{i, j}\right)},} \\
{\left[\mathbf{F}_{\mathrm{RF}}^{\text {cent }}\right]_{i, j}=\frac{1}{\sqrt{N_{\mathrm{t}}}} e^{j \measuredangle\left(\left[\mathbf{V}_{\text {cent }}\left(:, 1: N_{\mathrm{RF}}\right)\right]_{i, j}\right)} .}
\end{gathered}
$$

The digital TPC matrices $\mathbf{F}_{\mathrm{BB}}[k]$ and $\mathbf{F}_{\mathrm{BB}}^{\text {cent }}[k]$ are constructed according to (16). Fig. 4 presents the corresponding simulation results that AARG also exhibits an increasing trend as the bandwidth and $N_{\mathrm{s}}$ increase. When $N_{\mathrm{s}}=3$ and the bandwidth is $4 \mathrm{GHz}$, the average performance gap is higher than 10 $\mathrm{bps} / \mathrm{Hz}$. Therefore, schemes that assume common subspaces over all subcarriers cannot achieve satisfactory performance for high bandwidths. 
Based on the above observations, in the next two sections, we propose several new analog TPC design methods by exploiting the full CSI across all frequencies and from the array-vectors' perspectives.

\section{ANALOG TPC BAsed ON Full CSI}

In this section, several analog TPC schemes are proposed based on full CSI. The core of these methods is that we project all subcarrier channel to the central subcarrier channel and regard the total channel matrix as a non-sparse single "frequency-flat" channel so that we can construct the analog TPC matrix for all subcarriers simultaneously. This is a brandnew wideband hybrid TPC design perspective.

\section{A. Mean Channel Matrix (MCM)}

The most straightforward method is to utilize the mean channel matrix across all frequencies, which can be formulated as:

$$
\begin{aligned}
\overline{\mathbf{H}} & =\frac{1}{K} \sum_{k=1}^{K} \mathbf{H}[k] \\
& =\frac{\gamma}{K} \sum_{k=1}^{K} \sum_{m=1}^{N_{\mathrm{cl}}} \sum_{n=1}^{N_{\text {ray }}} \alpha_{m, n} \beta_{m, n, k} \mathbf{a}_{\mathrm{r}}\left(\phi_{m, n, k}\right) \mathbf{a}_{\mathrm{t}}^{*}\left(\varphi_{m, n, k}\right) \\
& =\frac{\gamma}{K} \sum_{m=1}^{N_{\text {cl }}} \sum_{p=1}^{K N_{\text {ray }}} \alpha_{m, n} \beta_{m, p} \mathbf{a}_{\mathrm{r}}\left(\bar{\phi}_{m, p}\right) \mathbf{a}_{\mathrm{t}}^{*}\left(\bar{\varphi}_{m, p}\right),
\end{aligned}
$$

where

$$
\bar{\varphi}_{m, p}=\frac{f_{\mathrm{c}}}{\mathrm{c}} d \sin \left(\bar{\vartheta}_{m, p}\right)=\frac{1}{2} \sin \left(\bar{\vartheta}_{m, p}\right),
$$

and

$$
\bar{\vartheta}_{m, p}=\vartheta_{m,\left\lceil\frac{p}{K}\right\rceil, p-\left\lfloor\frac{p}{K}\right\rfloor K}
$$

can be obtained according to (21). Furthermore, $\bar{\phi}_{m, p}$ in (29) have analogous definitions. Note that the MCM $\overline{\mathbf{H}}$ is frequency-independent and the difference between subcarriers is mainly reflected by their different physical steering angles. Based on $\overline{\mathbf{H}}$, the analog TPC matrix can be constructed by

$$
\widetilde{\mathbf{F}}_{\mathrm{RF}}^{\mathrm{MCM}}=\overline{\mathbf{V}}\left(:, 1: N_{\mathrm{RF}}^{\mathrm{t}}\right),
$$

where $\overline{\mathbf{V}}$ is the right singular vector obtained by

$$
\overline{\mathbf{H}}=\overline{\mathbf{U}} \overline{\mathbf{\Sigma}} \overline{\mathbf{V}}^{*} .
$$

Finally, similar to [21]-[23], the phase extraction operation is utilized to meet the CM constraint of the analog TPC matrix by

$$
\left[\mathbf{F}_{\mathrm{RF}}^{\mathrm{MCM}}\right]_{i, j}=\frac{1}{\sqrt{N_{\mathrm{t}}}} e^{j \measuredangle\left(\left[\widetilde{\mathbf{F}}_{\mathrm{RF}}^{\mathrm{MCM}}\right]_{i, j}\right)} .
$$

Although this MCM based scheme provides a simple and straightforward analog TPC solution for all subcarriers, it does not consider the correlation between different subcarriers and between different paths, even if the subspace distance of the diverse subcarriers may be high under a wide bandwidth and even if the number of paths is limited. However, it is anticipated that the MCM based scheme is only capable of achieving a moderate performance, as shown by our simulation results. Nevertheless, this scheme provides a valuable benchmark, which characterise the performance we can achieve when we simply regard the sparse frequency-selective channel to be a non-sparse frequency-flat one and do not take advantage of the subcarrier's and paths' correlation information.

\section{B. Mean Channel Covariance Matrix (MCCM)}

In this proposed approach, the mean channel covariance matrix is capitalized on for constructing the analog TPC, which is time-invariant even in the face of time-varying channel parameters. As an added benefit, the noise level is also reduced by the averaging operation.

Let us first recall the achievable sum rate from (12), which obeys the following inequality

$$
\begin{aligned}
R_{\mathrm{sum}} & \stackrel{(a)}{\leq} \frac{1}{K} \sum_{k=1}^{K} \log _{2}\left(\left|\mathbf{I}_{N_{\mathrm{r}}}+\frac{1}{\sigma_{n}^{2}}\left(\mathbf{H}[k] \mathbf{F}_{\mathrm{RF}} \mathbf{F}_{\mathrm{RF}}^{*} \mathbf{H}^{*}[k]\right)\right|\right) \\
& \stackrel{(b)}{=} \frac{1}{K} \sum_{k=1}^{K} \log _{2}\left(\left|\mathbf{I}_{N_{\mathrm{RF}}}+\frac{1}{\sigma_{n}^{2}}\left(\mathbf{F}_{\mathrm{RF}}^{*} \mathbf{H}^{*}[k] \mathbf{H}[k] \mathbf{F}_{\mathrm{RF}}\right)\right|\right) \\
& \stackrel{(c)}{\leq} \log _{2}\left(\left|\mathbf{I}_{N_{\mathrm{RF}}}+\frac{1}{\sigma_{n}^{2} K}\left(\mathbf{F}_{\mathrm{RF}}^{*} \sum_{k=1}^{K} \mathbf{H}^{*}[k] \mathbf{H}[k] \mathbf{F}_{\mathrm{RF}}\right)\right|\right),
\end{aligned}
$$

where (a) can be readily obtained by regarding $\mathbf{H}[k] \mathbf{F}_{\mathrm{RF}}$ as the equivalent channel matrix, (b) arises from the fact that $|\mathbf{I}+\mathbf{A B}|=|\mathbf{I}+\mathbf{B A}|$ by defining $\mathbf{A}=\mathbf{H}[k] \mathbf{F}_{\mathrm{RF}}$ and $\mathbf{B}=\mathbf{F}_{\mathrm{RF}}^{*} \mathbf{H}_{u}^{*}[k]$ and (c) is derived by employing Jensen's inequality. The equality in (c) holds if and only if $\mathbf{F}_{\mathrm{BB}}[k]$ is a unitary matrix and the equality in (c) holds, when we have $\mathbf{H}[1]=\mathbf{H}[2]=\ldots=\mathbf{H}[K]$, which means that each subcarrier has the same subspace. Although the equalities in (35) may not hold for a large bandwidth, the analog TPC can be still constructed by maximizing this upper bound similar as [29]. Accordingly, the optimization problem (19) is relaxed as

$$
\begin{gathered}
\left(\mathbf{F}_{\mathrm{RF}}^{\mathrm{opt}}\right)=\arg \max \log _{2}\left(\left|\mathbf{I}_{N_{\mathrm{RF}}}+\frac{1}{\sigma_{n}^{2} K}\left(\mathbf{F}_{\mathrm{RF}}^{*} \mathbf{R} \mathbf{F}_{\mathrm{RF}}\right)\right|\right), \\
\text { s.t. }\left|\left[\mathbf{F}_{\mathrm{RF}}\right]_{i, j}\right|^{2}=\frac{1}{N_{\mathrm{t}}}, \forall i, j,
\end{gathered}
$$

where the mean channel covariance matrix is given by

$$
\begin{aligned}
\mathbf{R} & =\frac{1}{K} \sum_{k=1}^{K} \mathbf{H}^{*}[k] \mathbf{H}[k] \\
& =\frac{1}{K} \sum_{k=1}^{K} \mathbf{V}[k] \boldsymbol{\Sigma}^{*}[k] \mathbf{\Sigma}[k] \mathbf{V}^{*}[k] \\
& =\frac{1}{K} \sum_{k=1}^{K} \mathbf{V}[k]\left[\begin{array}{c}
\lambda_{1, k} \\
\lambda_{2, k} \\
\ddots \\
\lambda_{N_{\text {path }}, k} \\
\mathbf{0}_{N_{\mathrm{t}}-N_{\text {path }}}
\end{array}\right] \mathbf{V}^{*}[k],
\end{aligned}
$$

where $N_{\text {path }}=N_{\text {cl }} N_{\text {ray }}$ is the total number of paths. Note that, although all the right subspaces of each subcarrier are utilized for calculating the channel covariance, they are weighted 
by the corresponding eigenvalues. Since the mmWave channel of each subcarrier is sparse, only the first $N_{\text {path }}$ eigenvalues are non-zero, which means there is very little power leakage to unused subspaces, even when $N_{\mathrm{RF}}^{\mathrm{t}}<N_{\text {path }}$. Therefore, the analog TPC can be obtained by extracting the phases of the first $N_{\mathrm{RF}}^{\mathrm{t}}$ eigenvectors of $\mathbf{R}=\mathbf{V}_{\mathrm{R}} \boldsymbol{\Sigma}_{\mathrm{R}} \mathbf{V}_{\mathrm{R}}^{*}$ similarly to (34).

Compared to the MCM based scheme, this MCCM aided arrangement better exploits the correlation between both the paths and the subcarries. Moreover, the receiver subspaces, which are also affected by beam squint, are firstly removed before the averaging operation. Therefore, the MCCM based scheme has the potential to improve the achievable rate.

\section{Generalized Extrinsic Mean of the Subspaces (GEMS)}

The third scheme is based on the extrinsic mean of the subspace motivated by [31]. However, the authors of [31] only consider two special cases, where $f_{\mathrm{s}}=97 \mathrm{MHz}, f_{\mathrm{c}}=28$ $\mathrm{GHz}$ and $f_{\mathrm{s}}=1.7 \mathrm{GHz}, f_{\mathrm{c}}=60 \mathrm{GHz}$. Furthermore, they assume $N_{\mathrm{RF}}^{\mathrm{t}}=N_{\text {path }}=N_{\mathrm{s}}$. Therefore, in this subsection, we extend the extrinsic mean of the subspace based method to more general scenarios, where both $N_{\text {path }}$ and $N_{\mathrm{RF}}$ are arbitrary. Additionally, we adopt a large range of bandwidths. According to (24), the extrinsic mean of the subspaces can be written as

$$
\mathbf{R}_{\mathrm{EMS}}=\frac{1}{K} \sum_{k=1}^{K}\left(\mathbf{V}\left(:, 1: N_{\text {path }}\right)[k]\right)\left(\mathbf{V}\left(:, 1: N_{\text {path }}\right)[k]\right)^{*} .
$$

Note that the dimension of the analog TPC matrix is $\left(N_{\mathrm{t}} \times\right.$ $N_{\mathrm{RF}}^{\mathrm{t}}$ ). When $N_{\mathrm{RF}}^{\mathrm{t}}<N_{\text {path }}$, power will leak to the unused subspaces. Moreover, note that the power is equally shared by each right singular vector in (38), which may intensify the effect of power leakage. Therefore, we propose the GEMS technique for alleviating the power leakage, which is given by

$$
\mathbf{R}_{\mathrm{GEMS}}=\frac{1}{K} \sum_{k=1}^{K}\left(\mathbf{V}\left(:, 1: N_{\mathrm{min}}\right)[k]\right)\left(\mathbf{V}\left(:, 1: N_{\min }\right)[k]\right)^{*}
$$

where $N_{\min }=\min \left(N_{\mathrm{RF}}^{\mathrm{t}}, N_{\text {path }}\right)$ is the rank of the effective channel matrix after analog TPC. Accordingly, the analog TPC matrix can be constructed by extracting the phases of the first $N_{\mathrm{RF}}^{\mathrm{t}}$ eigenvectors of $\mathbf{R}_{\mathrm{GEMS}}$. Then, upon comparing $\mathbf{R}_{\text {GEMS }}$ and $\mathbf{R}$, it can be observed that the main difference is the sum weights of the singular vectors $\mathbf{V}[k]$. Due to the extremely complex relationships between the subspaces of different subcarriers, it is challenging to determine which summation method is better. Their performances may differ for different system parameters (such as the bandwidth) and the different channel parameters (such as the path gains and angles). Nevertheless, the simulation results to be provided in Section VI will show that both the MCCM and the GEMS based hybrid TPC schemes are capable of achieving similar performance to the excessive-complexity manifold optimization based hybrid TPC scheme (will be introduced in Section VI). It may be an interesting future direction to determine the optimal weight of each singular vector of each subcarrier from a theoretical perspective, so as to obtain the optimal hybrid TPC scheme of the wideband scenario.

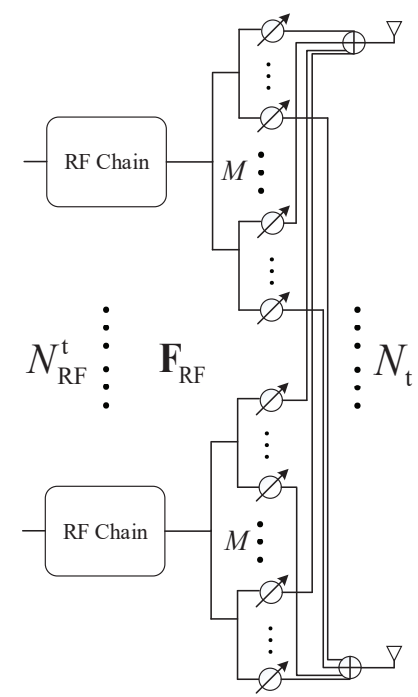

Fig. 5. Schematic of the multiple phase shifter-set based hybrid TPC architecture, where each RF chain is connected to antennas through $M$ sets of phase shifters.

\section{LOW-COMPLEXITy ANALOG TPC BASED ON ARRAY VECTORS}

Section VI introduced multiple full-CSI based analog TPC schemes, which are capable of achieving similar performance to the excessive-complexity manifold optimization based hybrid TPC scheme even for large bandwidths and antenna arrays. However, these schemes require SVD for each subcarrier channel, which may impose a high computational complexity, when the number of subcarriers is high (such as $K=128$ ). Therefore, in this section, another perspective is provided on designing the analog TPC matrices at an extremely lowcomplexity, which is purely based on the array-vectors. This idea is motivated by the following observations:

- When the number of antennas is much higher than the number of paths, each singular vector converges in chordal distance to an array response vector [6]. Therefore, the high-complexity SVD operation can be avoided.

- The items in the array-vectors satisfy the CM constraint so that the array vectors can be directly utilized for constructing the analog TPC.

- Since the phase/angle is the only variable in each array vector, it is easy to quantize the analog TPC matrix for facilitating a low feedback overhead based design.

- The angular coherence time is an order of magnitude longer than the traditional channel coherence time [21]. Therefore, the array-vector based analog TPC matrix may remain time-invariant over multiple channel coherence time intervals, which further reduces the complexity.

The key to our low-complexity analog TPC design is that of combining multiple array-vectors to construct wide beams so as to mitigate the effect of beam squint, which requires multiple sets of PSs to implement. The architecture of the multiple PS-set based analog TPC is shown in Fig. 5, where each RF chain is connected to antennas by $M \geq 1$ sets of PSs. However, there are also some constraints on our array-vector based schemes: 
- The number of RF chains required is set equal to the number of paths for simplifying the design, hence our array-vector based schemes cannot benefit from any further increase of the RF chains. Please note however that this constraint is not mandatory. When $N_{\mathrm{RF}}^{\mathrm{t}}>N_{\text {path}}$, the extra RF chains can be allocated to the paths having relatively high path-gains and be further exploited by subcarrier grouping, i.e., allocating multiple RF chains to a single path for constructing an analog TPC matrix for each subcarrier group. Having said that, it remains to be resolved, how to assign the extra RF chains, namely to which paths and which subcarriers, since it is challenging to obtain the corresponding optimal solution and may involve high-complexity computations.

- Multiple sets of PSs are required for each RF chain for combining multiple array-vectors of different subcarriers. Therefore, there is no need to meet the CM constraint for each entry of the analog TPC matrix, but this inevitably imposes additional hardware overhead.

- As we have shown in Section III, the mmWave channel having high bandwidths is not sparse when constructing the analog TPC. Therefore, angles of different paths have a higher probability of overlapping, which may impose a performance loss on the array-vectors based schemes.

In the following subsections, several array-vector combining schemes are elaborated on.

\section{A. Combining Array Vectors of All Subcarrier (AV-all)}

The first one relies on combining all array vectors over all subcarriers so that the power from all frequencies is captured, where $M=K$ and the number of $\mathrm{RF}$ chains required is $N_{\text {path }} K N_{\mathrm{t}}$. The analog TPC for the $n^{t h}$ path in the $m^{t h}$ cluster can be formulated as:

$$
\mathbf{f}_{\mathrm{RF}, m, n}^{\text {all }}=\sum_{k=1}^{K} \mathbf{a}_{\mathrm{t}}\left(\phi_{m, n, k}\right)
$$

and the full analog TPC matrix is given by

$$
\begin{aligned}
\mathbf{F}_{\mathrm{RF}}^{\text {all }} & =\left[\mathbf{f}_{\mathrm{RF}, 1,1}^{\text {all }}, \ldots, \mathbf{f}_{\mathrm{RF}, m, n}^{\text {all }}, \ldots, \mathbf{f}_{\mathrm{RF}, N_{\mathrm{cl}}, N_{\mathrm{ray}}}^{\text {all }}\right] \\
& =\sum_{k=1}^{K} \mathbf{A}_{\mathrm{t}}[k] .
\end{aligned}
$$

Note that array-vectors from different subcarriers are combined with equal weights, since the PS has to obey the CM constraint for each entry and can only change its phase. Therefore, the power on each RF chain is averaged across all subcarriers. Moreover, although the power from all frequencies are captured, which enables the analog TPC vector $\mathrm{f}_{\mathrm{RF}, m, n}^{\text {all }}$ to have the widest beam coverage, there is a potential power leakage at both sides and the inter-path interference also increases, especially when having a wide bandwidth, both of which may degrade the achievable rate.

\section{B. Selecting the Array-Vector of Single Subcarriers single)}

$(A V-$

Alternatively, in this subsection, the scheme that only chooses the array-vector of the central subcarrier as the analog TPC

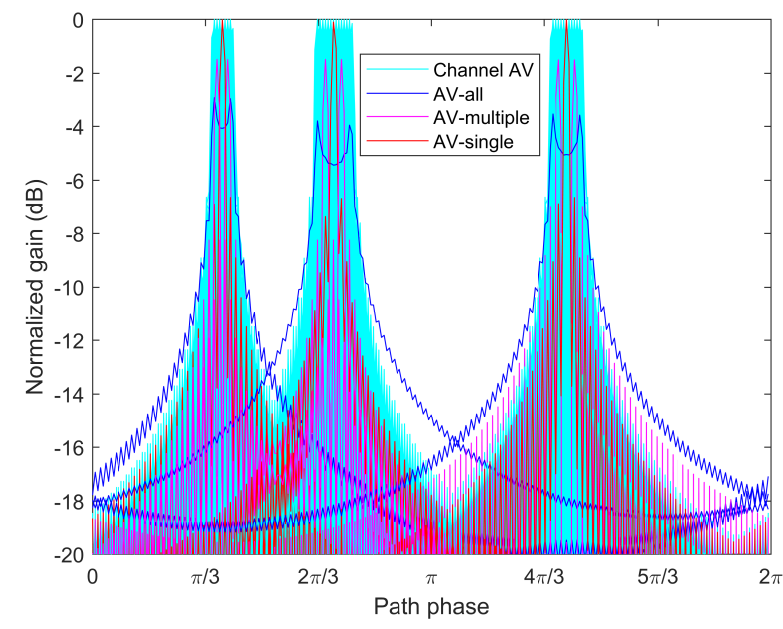

Fig. 6. Normalized gain for $4 \mathrm{GHz}$ bandwidth, and for $N_{\mathrm{t}}=128, K=128$, $N_{\mathrm{cl}}=3, N_{\text {ray }}=1$

is proposed, which has the narrowest beam coverage but the highest beam gain for the central subcarrier. In this scheme, $M=1$ and the analog TPC for the $n^{t h}$ path in the $m^{t h}$ cluster is formulated as:

$$
\begin{gathered}
\mathbf{f}_{\mathrm{RF}, m, n}^{\sin }=\mathbf{a}_{\mathrm{t}}\left(\bar{\varphi}_{m, n}\right), \\
\mathbf{F}_{\mathrm{RF}}^{\sin }=\left[\mathbf{f}_{\mathrm{RF}, 1,1}^{\mathrm{sin}}, \ldots, \mathbf{f}_{\mathrm{RF}, m, n}^{\sin }, \ldots, \mathbf{f}_{\mathrm{RF}, N_{\mathrm{cl}}, N_{\mathrm{ray}}}^{\sin }\right] .
\end{gathered}
$$

According to Fig. 3, when the bandwidth employed is not excessive, the chordal distance between subspaces of different subcarriers is relatively small. Therefore, the AV-single scheme of this section is capable of achieving good performance. Another benefit is that the computational complexity of the $\mathrm{AV}$-single scheme is the lowest among all and it is easy for it to assign the RF chains when $N_{\mathrm{RF}}^{\mathrm{t}} \leq N_{\text {path }}$. Explicitly, this can be achieved by selecting the $N_{\mathrm{RF}}$ array-vectors corresponding to the max $N_{\mathrm{RF}}$ path gains. However, the obvious disadvantage of the AV-single scheme is that the beam gains for the subcarriers that are relatively far from the central frequency are comparatively low. Hence the system performance is degraded.

\section{Combining Array-Vectors of Multiple Subcarriers (AV- multiple)}

Bearing in mind that there is a trade-off between the beam width and beam gain since the power of an array-vector is normalized, in this section, we combine multiple subcarriers for attaining the best possible performance in the family of array-vector based schemes. The corresponding analog TPC matrix may be written as

$$
\begin{aligned}
\mathbf{F}_{\mathrm{RF}}^{\mathrm{mul}} & =\left[\mathbf{f}_{\mathrm{RF}, 1,1}^{\mathrm{mul}}, \ldots, \mathbf{f}_{\mathrm{RF}, m, n}^{\mathrm{mul}}, \ldots, \mathbf{f}_{\mathrm{RF}, N_{\mathrm{cl}}, N_{\mathrm{ray}}}^{\mathrm{mul}}\right] \\
& =\sum_{k \in \mathcal{K}_{\mathrm{mul}}} \mathbf{A}_{\mathrm{t}}[k],
\end{aligned}
$$

where $\mathbf{f}_{\mathrm{RF}, m, n}^{\mathrm{mul}}$ is the analog TPC vector for the $n^{\text {th }}$ path in the $m^{\text {th }}$ cluster given by:

$$
\mathbf{f}_{\mathrm{RF}, m, n}^{\mathrm{mul}}=\sum_{k \in \mathcal{K}_{\mathrm{mul}}} \mathbf{a}_{\mathrm{t}}\left(\phi_{m, n, k}\right),
$$


TABLE II

SYSTEM PARAMETERS FOR SIMULATION.

\begin{tabular}{|l|l|}
\hline Parameters & Values \\
\hline Number of subcarriers $K$ & 128 \\
\hline Central carrier frequency $f_{\mathrm{c}}$ & $28 \mathrm{GHz}$ \\
\hline Bandwidth $f_{\mathrm{s}}$ & $0.3 \sim 4 \mathrm{GHz}$ \\
\hline Number of antennas & $\begin{array}{l}N_{\mathrm{t}}=16 \sim 160 \\
N_{\mathrm{r}}=16\end{array}$ \\
\hline Number of RF chains $N_{\mathrm{RF}}^{\mathrm{t}}$ & $3 \sim 12$ \\
\hline Number of data streams $N_{\mathrm{s}}$ & $1 \sim 6$ \\
\hline Number of paths [6], [21] & $\begin{array}{l}N_{\mathrm{cl}}=3 \\
N_{\mathrm{ray}}=2\end{array}$ \\
\hline
\end{tabular}

and $\mathcal{K}_{\text {mul }}$ is the selected subcarrier index set. Note that when $\mathcal{K}_{\text {mul }}=\left\{\frac{K+1}{2}\right\}$, (44) and (45) are the same as (42) and (43). When $\mathcal{K}_{\text {mul }}=\{1,2, \ldots, K\}$ i.e., the array-vectors from all subcarriers are selected, (44) and (45) become identical to (40) and (41). Combining the array-vectors of more subcarriers for one path corresponds to constructing a wider beam having lower peak beam gain, whilst utilizing array-vectors of a smaller number of subcarriers results in a narrower beam having higher peak beam gain. However, it is challenging to obtain the optimal subcarrier indexes, since high-complexity exhaustive search is required for evaluating all combinations and the total number of subcarriers is usually high, such as 128 , 256, etc. [31]. Fig. 6 shows the normalized gain of the analog TPC vectors constructed by the AV-all, $\mathrm{AV}$-single and $\mathrm{AV}$-multiple schemes, where $\mathcal{K}_{\text {mul }}$ is set to $\mathcal{K}_{\text {mul }}=\left\{\frac{K}{4}, \frac{3 K}{4}\right\}$. Observe that the analog vector constructed by the AV-all scheme has the maximum beam width but also the highest power leakage. By contrast, the AV-single scheme has the highest peak beam gain but the narrowest beam width. The AV-multiple scheme strikes a compelling compromise, which may result in the best achievable rate.

\section{Simulation Results}

In this section, extensive numerical simulations are presented for characterizing the performance of the proposed hybrid TPC schemes in our wideband mmWave MIMO system. Specifically, the proposed full-CSI based hybrid TPC schemes of Section IV are respectively marked as 'MCM', 'MCCM' and 'GEMS', while the low-complexity array-vector based schemes are marked as 'AV-all', 'AV-multiple' and 'AV-single'. ULAs are used at both the BS and MSs with $\lambda / 2$ elementspacing, where $\lambda$ is the wavelength of the central frequency. The main system parameters are summarized in Table II. Please note that the bandwidth $f_{\mathrm{s}}$ varies from $0.3 \mathrm{GHz}$ to $4 \mathrm{GHz}$. Therefore, the range of the normalized bandwidth $f_{\mathrm{s}} / f_{\mathrm{c}}$ is approximately $1.07 \% \sim 14.29 \%$. Moreover, the azimuth AOAs and AODs obey the Laplacian distribution with uniformly distributed mean angles within $(0,2 \pi]$ and angular spreads of $10^{\circ}$. The path delays are assumed to follow the uniform distribution as $\tau_{m, n} \sim \mathcal{U}(0,20 \mathrm{~ns})$ and $\max \tau_{m, n}=20 \mathrm{~ns}$,

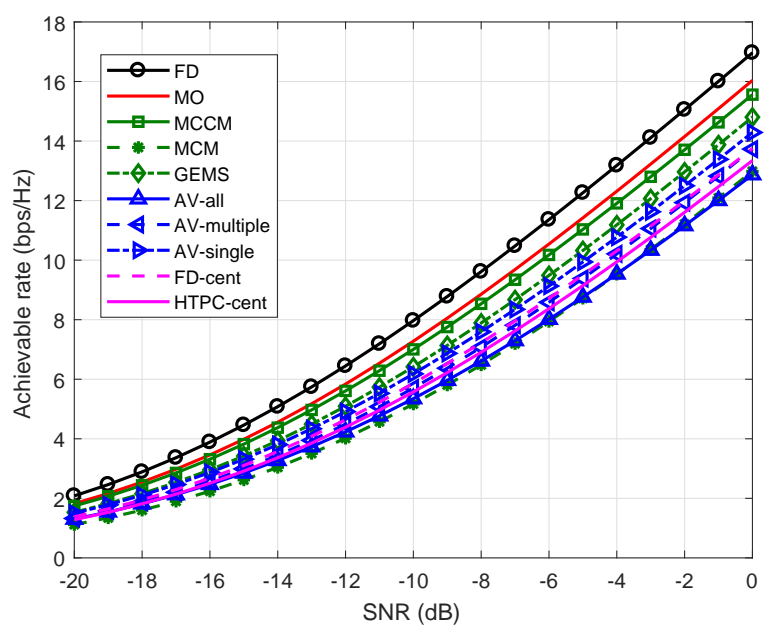

Fig. 7. The performance of the proposed full-CSI and array-vector based hybrid TPC schemes for different SNRs, where $N_{\mathrm{t}}=128, N_{\mathrm{r}}=16, f_{\mathrm{s}}=1$ $\mathrm{GHz}, N_{\mathrm{s}}=3$ and $N_{\mathrm{RF}}^{\mathrm{t}}=2 N_{\mathrm{s}}$.

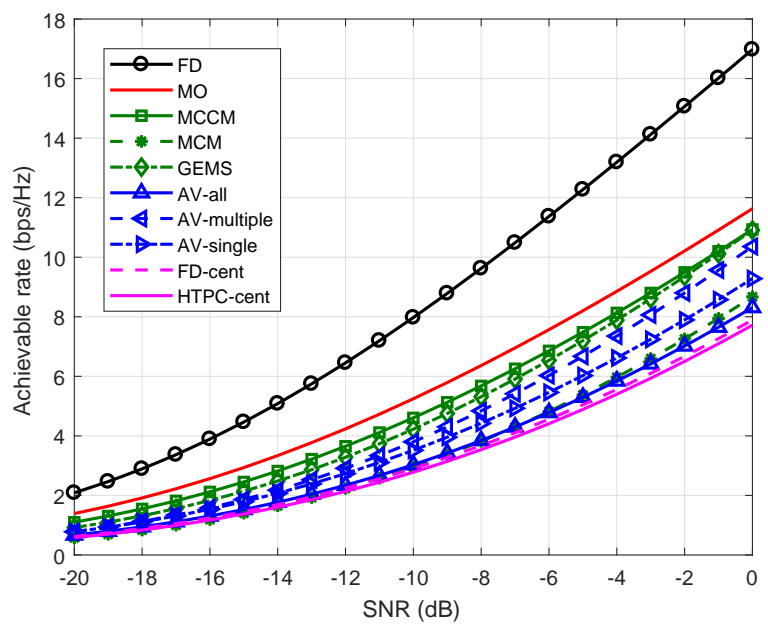

Fig. 8. The performance of the proposed full-CSI and array-vector based hybrid TPC schemes for different SNRs, where $N_{\mathrm{t}}=128, N_{\mathrm{r}}=16, f_{\mathrm{s}}=4$ $\mathrm{GHz}, N_{\mathrm{s}}=3$ and $N_{\mathrm{RF}}^{\mathrm{t}}=2 N_{\mathrm{s}}$.

while the path gains obey $\alpha_{m, n} \sim \mathcal{C N}\left(0, \sigma_{\alpha}^{2}\right)$ with $\sigma_{\alpha}^{2}=1$ [32]. Finally, the signal-to-noise ratio (SNR) is defined as $\frac{P_{t}}{\sigma_{n}^{2}}$.

Benchmark. Firstly, The fully digital TPC scheme that only utilizes the CSI of the central frequency shown in (22) is adopted as the benchmark (marked as 'FD-cent'), which represents the optimal precoder without taking the beam squint into account. The corresponding hybrid TPC scheme is marked as 'HTPC-cent', in which the analog TPC matrix is given by the phase extraction operation and the digital TPC matrix can be similarly constructed according to Lemma 1 by substituting $\mathbf{H}_{\text {cent }}[k]$ into (14). Secondly, we adopt the high-complexity traditional frequency-dependent fully digital scheme (marked as 'FD') as the upper bound, which can be readily constructed by the right singular vectors of $\mathbf{H}[k]$ seen in (24). Finally, the high-complexity manifold optimization based hybrid TPC method (marked as 'MO') is also used as a benchmark, which can be regarded as the optimal hybrid TPC scheme, 


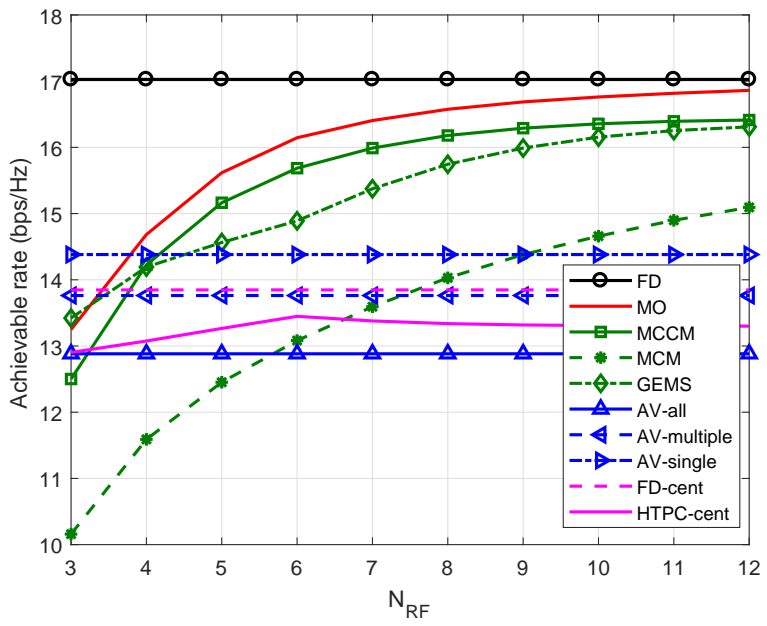

Fig. 9. The performance of the proposed full-CSI and array-vector based hybrid TPC schemes for different numbers of RF chains, where $N_{\mathrm{t}}=128$, $N_{\mathrm{r}}=16, f_{\mathrm{s}}=1 \mathrm{GHz}, N_{\mathrm{s}}=3$ and $\mathrm{SNR}=0 \mathrm{~dB}$.

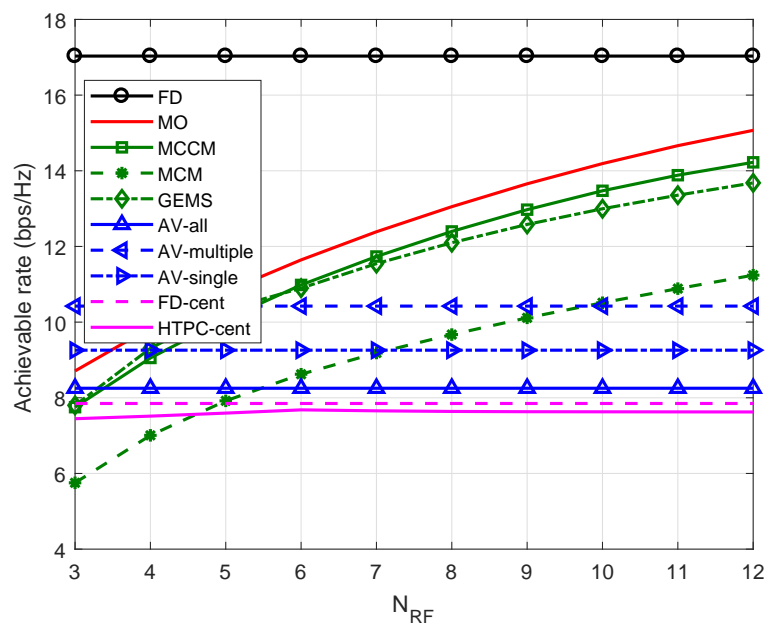

Fig. 10. The performance of the proposed full-CSI and array-vector based hybrid TPC schemes for different numbers of RF chains, where $N_{\mathrm{t}}=128$, $N_{\mathrm{r}}=16, f_{\mathrm{s}}=4 \mathrm{GHz}, N_{\mathrm{s}}=3$ and $\mathrm{SNR}=0 \mathrm{~dB}$.

when it achieves global optimality. Note that the MO scheme requires a high-complexity iterative process to converge, which is related to the iteration stopping criterion. In our simulations, in order to reduce the complexity but still attain a nearoptimal performance, the iteration stopping criterion is set to $|p(1)-p(2)| \leq 0.1$, where $p(1)$ and $p(2)$ are the cost function values in two consecutive iterations and can be calculated as $p(\cdot)=\sum_{k=1}^{K}\left\|\mathbf{F}_{\text {opt }}[k]-\mathbf{F}_{\mathrm{RF}}^{\mathrm{MO}} \mathbf{F}_{\mathrm{BB}}^{\mathrm{MO}}[k]\right\|_{F}^{2}$, in which $\mathbf{F}_{\mathrm{opt}}[k]$ is the optimal precoding matrix obtained by the frequency-dependent fully digital scheme. More details about the MO based hybrid TPC scheme can be found in [7].

In Fig. 7 and Fig. 8, we evaluate the achievable rate of the proposed full-CSI and array-vector based hybrid TPC schemes for different SNRs, where we have $N_{\mathrm{t}}=128, N_{\mathrm{r}}=16$, $N_{\mathrm{s}}=3, N_{\mathrm{RF}}^{\mathrm{t}}=2 N_{\mathrm{s}}, \mathcal{K}_{\mathrm{mul}}=\left\{\frac{K}{4}, \frac{3 K}{4}\right\}$ and the bandwidth is $1 \mathrm{GHz}$ and $4 \mathrm{GHz}$, respectively. We can observe that the

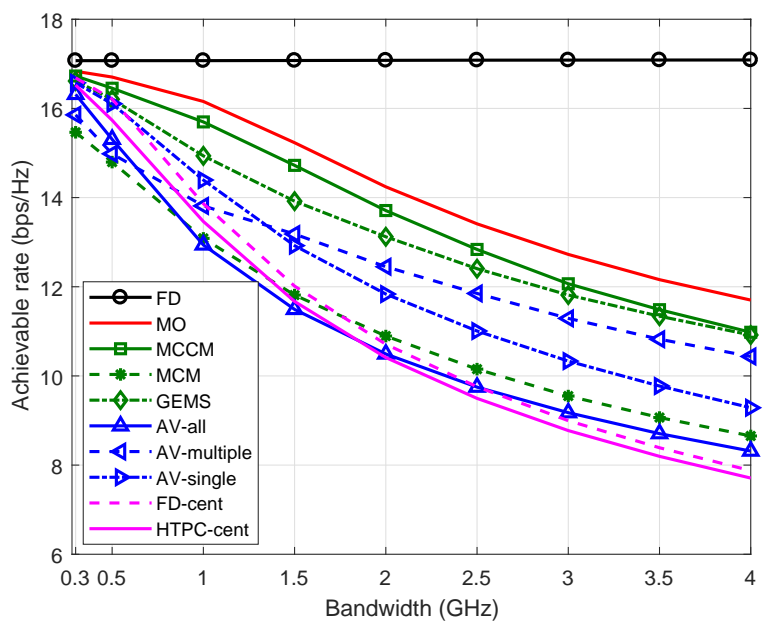

Fig. 11. The performance of the proposed full-CSI and array-vector based hybrid TPC schemes for different bandwidths, where $N_{\mathrm{t}}=128, N_{\mathrm{r}}=16$, $N_{\mathrm{S}}=3, N_{\mathrm{RF}}^{\mathrm{t}}=2 N_{\mathrm{s}}$ and $\mathrm{SNR}=0 \mathrm{~dB}$.

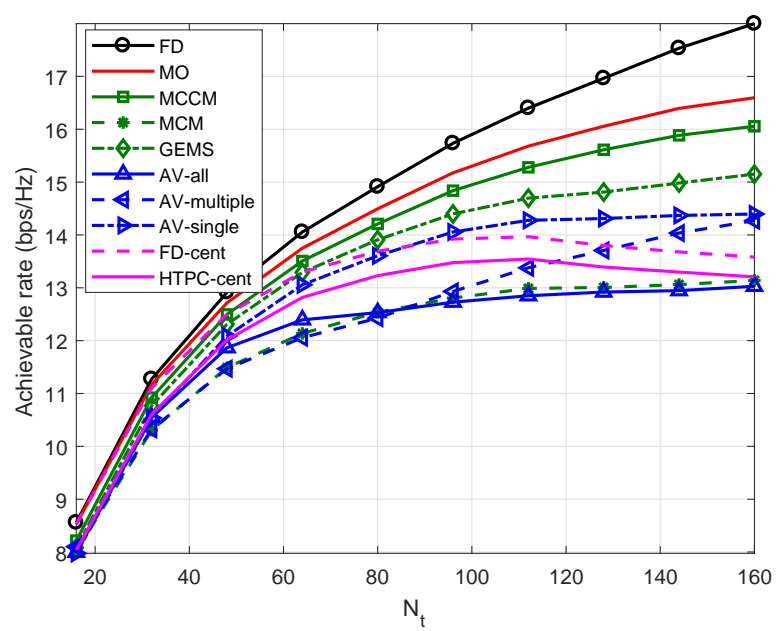

Fig. 12. The performance of the proposed full-CSI and array-vector based hybrid TPC schemes for different numbers of antennas at the BS, where $N_{\mathrm{r}}=16, f_{\mathrm{s}}=1 \mathrm{GHz}, N_{\mathrm{s}}=3, N_{\mathrm{RF}}^{\mathrm{t}}=2 N_{\mathrm{s}}$ and $\mathrm{SNR}=0 \mathrm{~dB}$.

proposed MCCM and GEMS based schemes perform always better than the hybrid scheme without considering the effect of beam squint and are even better than the corresponding fully digital scheme 'FD-cent', which validates the efficiency of our proposed frequency-independent analog TPC schemes. Furthermore, when $f_{\mathrm{s}}=1 \mathrm{GHz}$, it can be also observed that the proposed MCCM based scheme obtains nearly the same rate as the MO based scheme and has only approximately 1 $\mathrm{bps} / \mathrm{Hz}$ performance gap compared to the fully digital scheme. Moreover, Fig. 7 and Fig. 8 show that our proposed lowcomplexity array-vector based hybrid schemes are also capable of achieving good performance even for large bandwidths.

Fig. 9 and Fig. 10 compare the performance of different schemes for different numbers of RF chains, where we have $N_{\mathrm{t}}=128, N_{\mathrm{r}}=16, N_{\mathrm{s}}=3, \mathrm{SNR}=0 \mathrm{~dB}$ and the bandwidth is $1 \mathrm{GHz}$ and $4 \mathrm{GHz}$, respectively. Note that the number of RF chains for the low-complexity array-vector 


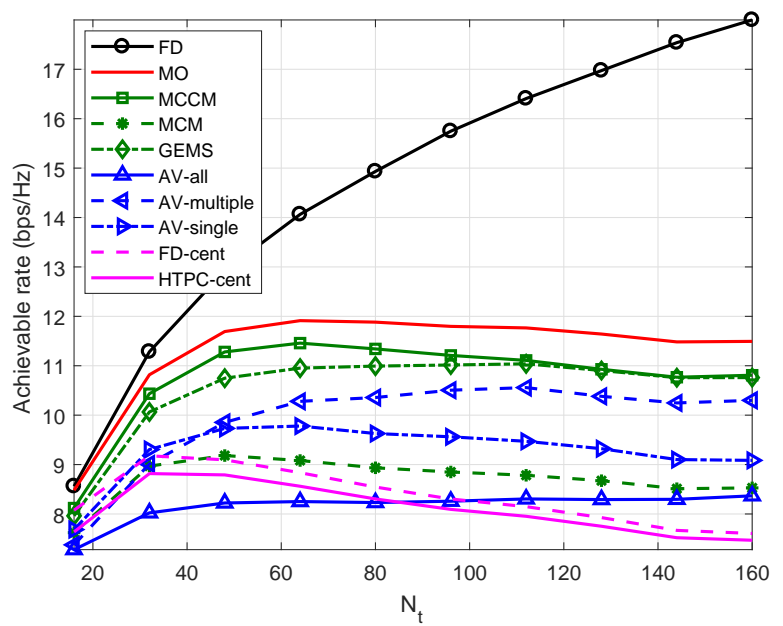

Fig. 13. The performance of the proposed full-CSI and array-vector based hybrid TPC schemes for different numbers of antennas at the BS, where $N_{\mathrm{r}}=16, f_{\mathrm{s}}=4 \mathrm{GHz}, N_{\mathrm{s}}=3, N_{\mathrm{RF}}^{\mathrm{t}}=2 N_{\mathrm{s}}$ and $\mathrm{SNR}=0 \mathrm{~dB}$.

based schemes is always set equal to the total number of paths, thus the achievable rate of the AV-all, AV-multiple and AV-single schemes remain unchanged, as $N_{\mathrm{RF}}^{\mathrm{t}}$ increases. We can observe that for $1 \mathrm{GHz}$ bandwidth, the MO based scheme achieves near-optimal performance, when applying $N_{\mathrm{RF}}^{\mathrm{t}}=3 N_{\mathrm{s}} \mathrm{RF}$ chains and the performance gaps w.r.t. our proposed MCCM and GEMS schemes are smaller than 1 bps $/ \mathrm{Hz}$. When $f_{\mathrm{s}}=4 \mathrm{GHz}$, the performance of the proposed MCCM, GEMS and MCM based schemes also increases with the number of RF chains and becomes better than that of the 'FD-cent' and 'HTPC-cent' schemes, which cannot benefit from the increased number of RF chains.

Fig. 11 illustrates the performance of different schemes for different bandwidths, where we have $N_{\mathrm{t}}=128, N_{\mathrm{r}}=16$, $N_{\mathrm{s}}=3, \mathrm{SNR}=0 \mathrm{~dB}$ and $N_{\mathrm{RF}}^{\mathrm{t}}=2 N_{\mathrm{s}}$. It can be observed that the achievable rates of all schemes, except for the frequencydependent fully digital scheme 'FD', exhibit a downward trend as the bandwidth increases. This because the effect of beam squint becomes more severe for wider bandwidths. Moreover, we observe that the proposed MCCM and GEMS schemes always achieve better performance than the FD-cent and HTPC-cent schemes, and the performance gaps increase with the bandwidth. Additionally, we also observe that upon applying small bandwidths, the AV-single scheme is the best among the array-vector based schemes. By contrast, the AVmultiple scheme becomes the best, when applying a wide bandwidth and all array-vector based schemes achieve better performance than the 'FD-cent' and 'HTPC-cent' schemes for $f_{\mathrm{s}} \geq 3 \mathrm{GHz}$.

In Fig. 12 and Fig. 13, we present the achievable rate against $N_{\mathrm{t}}$, where we have $N_{\mathrm{r}}=16, N_{\mathrm{s}}=3, N_{\mathrm{RF}}^{\mathrm{t}}=2 N_{\mathrm{s}}$, $\mathrm{SNR}=0 \mathrm{~dB}$ and $f_{\mathrm{s}}$ equals $1 \mathrm{GHz}$ and $4 \mathrm{GHz}$, respectively. We observe that the proposed MCCM and GEMS schemes achieve better performance than the 'FD-cent' and 'HTPCcent' schemes for different $N_{\mathrm{t}}$. Additionally, when applying a $1 \mathrm{GHz}$ bandwidth, all schemes exhibit an upward trend as the number of antennas increases. However, when increasing

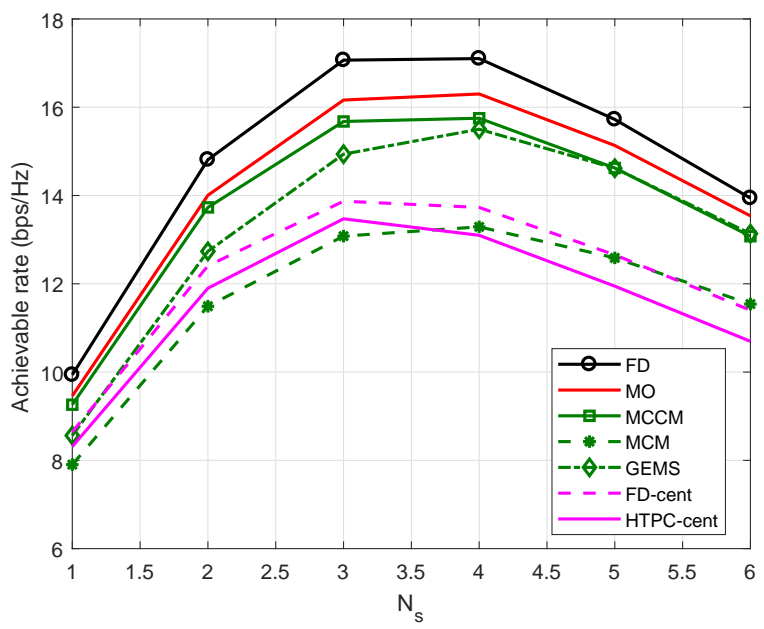

Fig. 14. The performance of the proposed full-CSI and array-vector based hybrid TPC schemes for different numbers of data streams, where $N_{\mathrm{t}}=128$, $N_{\mathrm{r}}=16, f_{\mathrm{s}}=1 \mathrm{GHz}, N_{\mathrm{RF}}^{\mathrm{t}}=2 N_{\mathrm{s}}$ and $\mathrm{SNR}=0 \mathrm{~dB}$.

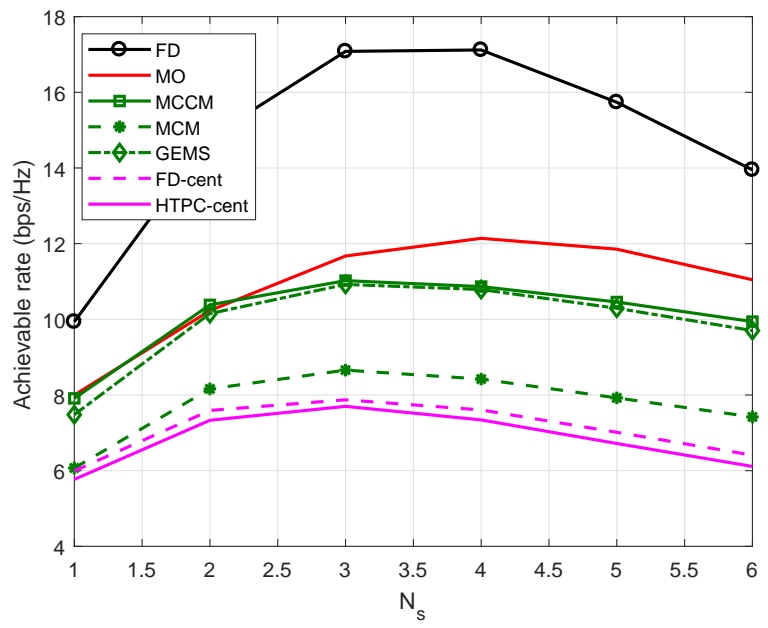

Fig. 15. The performance of the proposed full-CSI and array-vector based hybrid TPC schemes for different numbers of data streams, where $N_{\mathrm{t}}=128$, $N_{\mathrm{r}}=16, f_{\mathrm{s}}=4 \mathrm{GHz}, N_{\mathrm{RF}}^{\mathrm{t}}=2 N_{\mathrm{s}}$ and $\mathrm{SNR}=0 \mathrm{~dB}$.

the bandwidth to $4 \mathrm{GHz}$, the achievable rates of all schemes, except for the FD and AV-all schemes, are first increased and then start to gradually decay as $N_{\mathrm{t}}$ increases, which indicates that the performance increase benefits of having more antennas is lower than the performance degradation imposed by the beam squint. Therefore, it is vital to employ hybrid TPC schemes, which have taken the effect of beam squint into account for mmWave MIMO systems having a wide bandwidth and large antenna arrays.

Finally, in Fig. 14 and Fig. 15, we present the performance of different schemes for different numbers of data streams, where we have $N_{\mathrm{t}}=128, N_{\mathrm{r}}=16, N_{\mathrm{RF}}^{\mathrm{t}}=2 N_{\mathrm{s}}$, SNR = $0 \mathrm{~dB}$ and the bandwidth is $1 \mathrm{GHz}$ and $4 \mathrm{GHz}$, respectively. We observe that the achievable rate of all schemes is firstly increased then decreased as the number of data streams increases. This is because when $N_{\mathrm{s}}$ is small relative to the number of paths, transmitting more data steams can benefit 
from the multiplexing gains. However, when $N_{\mathrm{S}}$ approaches to $N_{\text {path }}$, all paths, including the path with low path gains, are utilized to transmit data streams, but the total power is equally allocated to all data streams. Moreover, it can be observed that when $f_{\mathrm{s}}=1 \mathrm{GHz}$, the proposed MCCM scheme achieves a performance close to that of the $\mathrm{MO}$ and 'FD' schemes. However, when $f_{\mathrm{s}}=4 \mathrm{GHz}$, the performance gap is increased to be higher than $5 \mathrm{bps} / \mathrm{Hz}$, which reveals the limitation of the phase shifter based hybrid architecture in wideband scenarios.

\section{CONCLUSIONS}

In this paper, we focused our attention on the challenging design of hybrid TPC matrices for wideband mmWave MIMO systems in the face severe beam squint. We firstly showed that the channel matrices of the different subcarriers possess distinct subspaces in case of high bandwidths. Based on our MCD and AARG metrics, we then showed that there will be huge performance losses, when employing traditional hybrid TPC schemes assuming common subspaces across all subcarriers. Accordingly, we proposed several full-CSI based hybrid TPC schemes from a new perspective by projecting all frequencies to the central frequency and then constructing common analog TPC matrices for all subcarriers, including the MCM, MCCM and GEMS based hybrid TPC schemes. Furthermore, we also proposed several low-complexity arrayvector based hybrid TPC schemes by combining the arrayvectors from single, multiple and all subcarriers to construct wide beams so as to mitigate the effect of beam squint. By using the high-complexity frequency-dependent fully digital TPC scheme and the MO based hybrid TPC scheme as the benchmarks, our extensive numerical simulations demonstrated that the proposed schemes are capable of achieving nearoptimal performance when the bandwidth approaches $0.5 \mathrm{GHz}$ and show obvious superiority over the traditional hybrid TPC scheme ignoring the beam squint.

Note that the frequency-independent phase shifter based analog architecture has inherent disadvantages when supporting large bandwidths, since the severe beam squint results in frequency-dependent beams. Our future follow-up work may consider combining the analog TPC design with chirp sequences [33] for constructing wide beams and for reducing the number of phase shifters required. Moreover, the true time delay solution [34] is also a promising hardware that can be introduced into the design of hybrid TPC, which is capable of controlling the delay so that the phase of the beam changes with the frequency to eliminate the squint phenomenon.

\section{REFERENCES}

[1] I. A. Hemadeh, K. Satyanarayana, M. El-Hajjar and L. Hanzo, "Millimeter-wave communications: physical channel models, design considerations, antenna constructions, and link-budget," IEEE Commun. Surveys Tuts., vol. 20, no. 2, pp. 870-913, 2nd Quart. 2018.

[2] Y. Chen, W. Liu, Z. Niu, Z. Feng, Q. Hu, T. Jiang, "Pervasive intelligent endogenous $6 \mathrm{G}$ wireless systems: prospects, theories and key technologies," Digit. Commun. Netw., vol. 6, no. 3, pp. 312-320, Aug. 2020.

[3] R. W. Heath, N. González-Prelcic, S. Rangan, W. Roh and A. M. Sayeed, "An overview of signal processing techniques for millimeter wave MIMO systems," IEEE J. Sel. Areas Commun., vol. 10, no. 3, pp. 436-453, Apr. 2016.
[4] O. E. Ayach, S. Rajagopal, S. Abu-Surra, Z. Pi, and R. W. Heath, Jr, "Spatially sparse precoding in millimeter wave MIMO systems," IEEE Trans. Wireless Commun., vol. 13, no. 3, pp. 1499-1513, Mar. 2014.

[5] K. Satyanarayana, M. El-Hajjar, P. H. Kuo, A. Mourad and L. Hanzo, "Dual-function hybrid beamforming and transmit diversity aided millimeter wave architecture," IEEE Trans. Veh. Technol., vol. 67, no. 3, pp. 2798-2803, Mar. 2018.

[6] Y. Chen, D. Chen, and T. Jiang, "Non-uniform quantization codebook based hybrid precoding to reduce feedback overhead in millimeter wave MIMO systems," IEEE Trans. Commun., vol. 67, no. 4, pp. 2779-2791, Apr. 2019

[7] X. Yu, J.-C. Shen, J. Zhang, and K. B. Letaief, "Alternating minimization algorithms for hybrid precoding in millimeter wave MIMO systems," IEEE J. Sel. Topics Signal Process., vol. 10, no. 3, pp. 485-500, Apr. 2016.

[8] W. Ni and X. Dong, "Hybrid block diagonalization for massive multiuser MIMO systems," IEEE Trans. Commun., vol. 64, no. 1, pp. 201-211, Jan. 2016

[9] C. E. Chen, "An iterative hybrid transceiver design algorithm for millimeter wave MIMO systems," IEEE Wireless Commun. Lett.,vol. 4, no. 3, pp. 285-288, Jun. 2015.

[10] X. Gao, L. Dai, C. Yuen, and Z. Wang, "Turbo-like beamforming based on tabu search algorithm for millimeter-wave massive MIMO systems,' IEEE Trans. Veh. Technol., vol. 65, no. 7, pp. 5731-5737, Jul. 2016.

[11] Y. Chen, D. Chen, Y. Tian, and T. Jiang, "Spatial lobes division based low complexity hybrid precoding and diversity combining for mmWave IoT systems," IEEE Internet Things J., vol. 6, no. 2, pp. 3228-3239, Apr. 2019

[12] C. Rusu, R. Méndez-Rial, N. González-Prelcic and R. W. Heath, "Low complexity hybrid precoding strategies for millimeter wave communication systems," IEEE Trans. Wireless Commun., vol. 15, no. 12, pp. 8380-8393, Dec. 2016.

[13] C. H. Chen, C. R. Tsai, Y. H. Liu, W. L. Hung and A. Y. Wu, "Compressive sensing (CS) assisted low-complexity beamspace hybrid precoding for millimeter-wave MIMO systems," IEEE Trans. Signal Process., vol. 65, no. 6, pp. 1412-1424, Mar. 2017.

[14] X. Gao, L. Dai, S. Han, C.-L. I, and R. W. Heath, "Energy-efficient hybrid analog and digital precoding for mmWave MIMO systems with large antenna arrays," IEEE J. Sel. Areas Commun., vol. 34, no. 4, pp. 998-1009, Apr. 2016.

[15] V. V. Ratnam, A. F. Molisch, O. Y. Bursalioglu, and H. C. Papadopoulos, "Hybrid beamforming with selection for multiuser massive MIMO systems," IEEE Trans. Signal Process., vol. 66, no. 15, pp. 4105-4120, Aug. 2018.

[16] D. Zhang, Y. Wang, X. Li, W. Xiang, "Hybridly-connected structure for hybrid beamforming in mmWave massive MIMO systems," IEEE Trans. Commun., vol. 66, no. 2, pp. 662-674, Feb. 2018.

[17] Y. Chen, D. Chen, T. Jiang, and L. Hanzo,"Millimeter-wave massive MIMO systems relying on generalized sub-array-connected hybrid precoding," IEEE Trans. Veh. Technol., vol. 68, no. 9, pp. 8940-8950, Sep. 2019.

[18] X. Gao, L. Dai, and A. M. Sayeed, "Low RF-complexity technologies to enable millimeter-wave MIMO with large antenna array for $5 \mathrm{G}$ wireless communications," IEEE Commun. Mag., vol. 56, no. 4, pp. 211-217, Apr. 2018.

[19] S. Park, A. Alkhateeb, and R. W. Heath, "Dynamic subarrays for hybrid precoding in wideband mmWave MIMO systems," IEEE Trans. Wireless Commun., vol. 16, no. 5, pp. 2907-2920, May 2017.

[20] S. Park and R. W. Heath, "Spatial channel covariance estimation for the hybrid MIMO architecture: a compressive sensing based approach," IEEE Trans. Wireless. Commun., vol. 17, no. 12, pp. 8047-8062, Dec. 2018.

[21] Y. Chen, D. Chen, T. Jiang, and L. Hanzo,"Channel-covariance and angle-of-departure aided hybrid precoding for wideband multiuser millimeter wave MIMO systems," IEEE Trans. Commun., vol. 67, no. 12, pp. 8315-8328, Dec. 2019.

[22] A. Alkhateeb and R. W. Heath, "Frequency selective hybrid precoding for limited feedback millimeter wave systems," IEEE Trans. Commun., vol. 64, no. 5, pp. 1801-1818, May 2016.

[23] J. P. González-Coma, J. Rodríguez-Fernández, N. González-Prelcic, L. Castedo and R. W. Heath, "Channel estimation and hybrid precoding for frequency selective multiuser mmWave MIMO systems," IEEE J. Sel. Top. Signal Process., vol. 12, no. 2, pp. 353-367, May 2018.

[24] Y. P. Lin, "Hybrid MIMO-OFDM beamforming for wideband mmwave channels without instantaneous feedback," IEEE Trans. Signal Process., vol. 66 , no. 19 , pp. 5142-5151, Oct. 2018. 
[25] B. Wang, F. Gao, S. Jin, H. Lin, G. Y. Li, S. Sun, and T. S. Rappaport, "Spatial-wideband effect in massive MIMO with application in mmWave systems," IEEE Commun. Mag., vol. 56, no. 12, pp. 134-141, Dec. 2018.

[26] B. Wang, F. Gao, S. Jin, H. Lin and G. Y. Li, "Spatial- and frequencywideband effects in millimeter-wave massive MIMO systems," IEEE Trans. Signal Process., vol. 66, no. 13, pp. 3393-3406, Jul. 2018.

[27] B. Wang, M. Jian, F. Gao, G. Y. Li and H. Lin, "Beam squint and channel estimation for wideband mmwave massive MIMO-OFDM systems," IEEE Trans. Signal Process., vol. 67, no. 23, pp. 5893-5908, Dec. 2019.

[28] B. Wang, X. Li, F. Gao and G. Y. Li, "Power leakage elimination for wideband mmwave massive MIMO-OFDM systems: an energy-focusing window approach," IEEE Trans. Signal Process., vol. 67, no. 21, pp. 5479-5494, Nov. 2019.

[29] W. Shen, X. Bu, X. Gao, C. Xing and L. Hanzo, "Beamspace precoding and beam selection for wideband millimeter-wave MIMO relying on lens antenna arrays," IEEE Trans. Signal Process., vol. 67, no. 24, pp. 6301-6313, Dec. 2019.

[30] B. Liu, W. Tan, H. Hu and H. Zhu, "Hybrid beamforming for mmWave MIMO-OFDM system with beam squint," in Proc. IEEE Int. Symp. Pers. Indoor Mobile Radio Commun. (PIMRC), Bologna, Italy, Sep. 2018, pp. $1422-1426$.

[31] K. Venugopal, N. González-Prelcic and R. W. Heath, "Optimal frequency-flat precoding for frequency-selective millimeter wave channels," IEEE Trans. Wireless. Commun., vol. 18, no. 11, pp. 5098-5112, Nov. 2019.

[32] X. Gao, L. Dai, S. Zhou, A. Sayeed, and L. Hanzo, "Beamspace channel estimation for wideband millimeter-wave MIMO with lens antenna array," in Proc. IEEE Int. Conf. Commun. (ICC), Kansas, US, May 2018, pp. 1-6.

[33] R. Peng and Y. Tian, "Robust wide-beam analog beamforming with inaccurate channel angular information," IEEE Commun. Lett., vol. 22, no. 3, pp. 638-641, Mar. 2018.

[34] R. Rotman, M. Tur, and L. Yaron, "True time delay in phased arrays," Proc. IEEE, vol. 104, no. 3, pp. 504-518, Mar. 2016. 